\title{
STUDYING THE WELFARE OF CHILDREN AT AN EARLY AGE IN THE SYSTEM OF INSTITUTIONAL CARE
}

\begin{tabular}{|c|c|}
\hline Dr. Sofiya Dermenc & $\begin{array}{l}\text { South-West University “Neofit Rilski” - Blagoevgrad, Bulgaria } \\
\text { E-mail: sofger@swu.bg }\end{array}$ \\
\hline A R T I C L E I N F O & $\begin{array}{l}\text { A B S T R A C T } \\
\text { This article discusses the need to develop a unified vision and strategy }\end{array}$ \\
\hline Original Research & for enhancing the welfare of children at an early age in the institutional care \\
\hline Received: March, 03.2019. & system in Bulgaria. The methodological basis for the research is the "Quality \\
\hline Revised: March, 28.2019. & Framework for Early Childhood Services" (QFECS) of the International \\
\hline $\begin{array}{l}\text { Accepted: April, } 09.2019 . \\
\text { doi:10.5937/ijcrsee1901095D }\end{array}$ & $\begin{array}{l}\text { Association Step-by-Step (ISSA). A diagnostic tool was developed that } \\
\text { explores the guiding principles of good practices in nine different priority } \\
\text { areas in three distinct groups in Bulgaria. It analyzes the state of early care }\end{array}$ \\
\hline UDK & and identifies the resources that ensure the quality of services during early \\
\hline $373.2(497.2)$ & childhood. \\
\hline
\end{tabular}

159.922 .7

Keywords:

welfare,

early childhood,

institutional care,

quality of services in nurseries.

(C) 2019 IJCRSEE. All rights reserved.

\section{INTRODUCTION}

As a matter of fact, the nurseries are defined as organizationally separate structures in which medical and other specialists carry out raising, educating and training children from three months to three years of age. In recent years, this definition has lost its relevance, given the results of early childhood and care research. The arguments in this regard are the research and the assertion of the role of prenatal development and the ever-increasing tendency that "the modern family, not only in an economic but also in a deep spiritual crisis, needs both socio-economic and professional psychological support “ (Tasevska, 2008: 12).

Contemporary research confirms that the child's future development is the first 1000 days of his life. According to their research relevance and relevance, actions are under way in Bulgaria aimed at:

- reassessing the status of crèches, respectively, of crèches in the system of institu-

Corresponding Author

Dr. Sofiya Dermendzhieva, South-West University "Neofit Rilski" - Blagoevgrad, Bulgaria

E-mail: sofger@swu.bg

\section{cc) (i) $\odot$}

This work is licensed under a Creative Commons Attribution - NonCommercial - NoDerivs 4.0. The article is published with Open Access at www.ijcrsee.com tional care;

- improving public services for early childhood development;

- clarifying the role of the institutions in developing a unified vision and strategy for improving the well-being of children at an early age;

- implementing cross-sectoral cooperation with a view to achieving a broad consensus on the implementation of a more integrated approach;

- approach in children's policies during this period.

An important input in this direction is the initiative of the ISSA, which develops and implements the "Quality Framework for Early Childhood Services" (QFECS).

The present study analyzes the achieved results and the effects of the "Quality Framework for Early Childhood Services" (QFECS) in three distinct groups of United Children's Establishments in Bulgaria.

\section{MATERIALS AND METHODS}

The "Quality Framework for Early Childhood Services" (QFECS) is based on the vision of the ISSA for a society in which families, communities and experts work together to enable each child to develop their full- potential.

The framework outlines the guiding 
principles of good practice in Nine different priority areas and details the indicators that describe ways to effectively implement them.

Its development is in line with other international frameworks and opinions, of which more important are:

- Early Childhood Education and Care (ECEC, 2014);

- Review of Policies and Practices for Monitoring and Quality Assessment of Early Learning and Development in Strong Start III (OECD, 2012);

- Early childhood education and care: to provide all children with the best start in life for tomorrow's world (EC-European Commission. 2011); CEF.

- Documents from UNESCO and UNI-

According to experts in the field of European early-school policy, the proposal to create a national pedagogical framework covering the period from birth to the start of schooling is characterized by high innovation and prospect, given the opportunity to provide "more effective management of the targeted system of social care in early and preschool age" (Mihova, 2012: 126).

Exploring the European standards towards children, Kaleynska in cludes the child welfare among the basic children's rights to be guaranteed by the Union policies (Kaleynska, 2014:125).

At a conceptual level, the Framework introduces new approaches to fostering care and well-being in early childhood, and focuses on updating policies on early childhood development.

At the instrumental level, its significance is expressed in the definition of Nine priority areas, the deployment of which ensures the parity of meeting the needs of all stakeholders: children, families and professionals in specialized institutions.

Recognizing the special needs of children from the birth to the age of three, the ISSA formulates specific principles and indicators in delivering high quality services to children of the elderly.

The framework contains potential in the context of the modern sustainable development paradigm, as it implies the idea that "education should not only be life-wide but also diversified, and learning intensively integrated into all major activities of life" (Dyankova, 2018a: 96). Detailed detailing of the principles characterizing the individual priority areas reflects the RCDC's potential for measuring the quality of services in early childhood.
At an applied level, the effectiveness of the Framework is contained in the substantiated description of specific indicators for recognizing the principles that basically define childcare at an early age.

Outlined practical expedience makes the Framework a reliable indicative tool, tracking logical consistency in identifying the results and effects of its application in social and institutional practices.

\subsection{Research program}

The research program has been deployed in four stages:

First stage: information, during which the following activities were carried out:

- meeting with the clergy teams of three clerical groups from the "Zvenche" Kindergarten and Kindergarten "Zname na mira", Vratsa and presenting the goals of the study;

- Establishing a timetable for conducting trainings with participants for the implementation of the "Quality Framework for Early Childhood Services" in the Task Force;

- Establishment of a timetable for visitor group visits for the purpose of monitoring and evaluating childcare provision at an early age after the training provided;

- Validation of the Early Childhood Quality Assessment tools: Monitoring Surveillance Daily of the Monitoring Expert and "Self-Assessment Daily Report" of the Jaslite Team Specialists.

Stage Two: A training course in which cluster specialists are involved in training modules.

Content-based trainings present the guiding principles of good practice in Nine Different Priority Areas for Quality of Service in Early Childhood and detail the ways for their effective implementation.

Third stage: approbing, in which the actual monitoring and assessment of the wellbeing of the children at an early age is carried out in accordance with the principles and priorities of the Framework.

Stage four: Final, analyzing the results and effects of RACC implementation. 


\section{RESULTS}

The analysis of the results and the evaluation of the effects is based on the data processing of the two main instruments:

- "Daily Surveillance Report", completed by the expert in the monitoring of the groups after the training;

- "Daily self-assessment report" after the training, reflecting the reflexion of the cluster teams on the guiding principles and ways to apply best practices in the 9 priority areas of the "Quality Framework for Early Childhood Services" (QFECS).

In substance, the described tools are identical, allowing their results to be compared and analyzed.

According to modern researchers, the observed correlations and discrepancies in the percentage ratio of the marked statements take into account trends typical of such studies:

- self-evaluation is a process in which the subjects unconsciously choose those degrees in the stated statements that rather reflect their "desirable" behavior;

- Surveillance is a process in which subjects dominate their focus on FACTS, reflecting difficulties in implementing "expectant" behavior (Dyankova, 2018b: 11).

Conditional compliance with these two trends is in the direction of subsequent analysis.

The Pilot Implementation of a "Quality Framework for Early Childhood Services" in three distinct groups in the municipality of Vratsa examines the state of early childhood care.

The focus of the study is defined in the principles and indicators of Nine priority areas.

The tables contain the quantitative values and percentage equivalents of the results of "Daily report self-assessment" of the members of the creche teams and "Daily report for observation" carried out by the expert monitoring.

Their discussion builds on the indicators that reveal the extent of resource outcomes that ensure the quality of services in early childhood.

\subsection{Priority area "Relationships"}

Table 1. Principle 1: Relationship with each child recognizes and values its uniqueness, competencies, personal style of communication, preferences and opinion.

\begin{tabular}{|c|c|c|c|c|c|c|}
\hline $\begin{array}{c}\text { Indicator } \\
1.1 .\end{array}$ & \multicolumn{6}{|c|}{$\begin{array}{l}\text { It responds to the child's signals } \\
\text { about his or her desires, interests, } \\
\text { and choices. }\end{array}$} \\
\hline Statements & \multicolumn{2}{|c|}{$\begin{array}{c}\text { Does not } \\
\text { apply }\end{array}$} & \multicolumn{2}{|c|}{$\begin{array}{l}\text { Sometim } \\
\text { es apply }\end{array}$} & \multicolumn{2}{|c|}{$\begin{array}{l}\text { Apply } \\
\text { always }\end{array}$} \\
\hline Results & \multicolumn{2}{|c|}{ Nr. $\%$} & \multicolumn{2}{|c|}{ Nr. $\%$} & \multicolumn{2}{|c|}{ Nr. $\%$} \\
\hline observations & 1 & 11.1 & & 33.3 & 5 & 55.56 \\
\hline self-assessment & 0 & 00.0 & 0 & 00.0 & 10 & 100.0 \\
\hline $\begin{array}{c}\text { Indicator } \\
1.2 .\end{array}$ & \multicolumn{6}{|c|}{$\begin{array}{l}\text { He meets the child in ways that } \\
\text { correspond to his temperament and } \\
\text { personality. }\end{array}$} \\
\hline Statements & \multicolumn{2}{|c|}{$\begin{array}{c}\text { Does not } \\
\text { apply }\end{array}$} & \multicolumn{2}{|c|}{$\begin{array}{l}\text { Sometim } \\
\text { es apply }\end{array}$} & \multicolumn{2}{|c|}{$\begin{array}{l}\text { Apply } \\
\text { always }\end{array}$} \\
\hline Results & \multicolumn{2}{|c|}{ Nr. $\%$} & \multicolumn{2}{|c|}{ Nr. $\%$} & \multicolumn{2}{|c|}{ Nr. $\%$} \\
\hline observations & 0 & 00.0 & 2 & 22.2 & 7 & 77.78 \\
\hline self-assess & 0 & 00.0 & & 00.0 & 10 & 100.0 \\
\hline $\begin{array}{c}\text { Indicator } \\
1.3 .\end{array}$ & \multicolumn{6}{|c|}{$\begin{array}{l}\text { It shows to the child that what he } \\
\text { says is listened to. }\end{array}$} \\
\hline Statements & \multirow{2}{*}{\multicolumn{2}{|c|}{$\begin{array}{l}\text { Does not } \\
\text { apply } \\
\text { Nr. } \%\end{array}$}} & \multicolumn{2}{|c|}{$\begin{array}{l}\text { Sometim } \\
\text { es apply }\end{array}$} & \multicolumn{2}{|c|}{$\begin{array}{l}\text { Apply } \\
\text { always }\end{array}$} \\
\hline Results & & & \multicolumn{2}{|c|}{ Nr. $\%$} & \multicolumn{2}{|c|}{ Nr. $\%$} \\
\hline observations & 2 & 22.2 & & 33.33 & 4 & 44.44 \\
\hline self-assessment & 0 & 00.0 & & 10.00 & 9 & 90.00 \\
\hline $\begin{array}{c}\text { Indicator } \\
1.4 .\end{array}$ & \multicolumn{6}{|c|}{$\begin{array}{l}\text { An understanding of the child's } \\
\text { preference for the tempo, time and } \\
\text { intensity of the speech, movements } \\
\text { and facial expressions is shown. }\end{array}$} \\
\hline Statements & \multicolumn{2}{|c|}{$\begin{array}{c}\text { Does not } \\
\text { apply }\end{array}$} & \multicolumn{2}{|c|}{$\begin{array}{l}\text { Sometim } \\
\text { es apply }\end{array}$} & \multicolumn{2}{|c|}{$\begin{array}{l}\text { Apply } \\
\text { always }\end{array}$} \\
\hline Results & \multicolumn{2}{|c|}{ Nr. $\%$} & \multicolumn{2}{|c|}{ Nr. $\%$} & \multicolumn{2}{|c|}{ Nr. $\%$} \\
\hline observations & 4 & 44.4 & 0 & 00.00 & 5 & 55.56 \\
\hline self-assessment & 0 & 00.0 & & 40.40 & 6 & 60.00 \\
\hline $\begin{array}{l}\text { Indicator } \\
1.5 .\end{array}$ & \multicolumn{6}{|c|}{$\begin{array}{l}\text { It interacts with the child, with } \\
\text { respect for him as an active } \\
\text { researcher and a capable participant. }\end{array}$} \\
\hline Statements & \multicolumn{2}{|c|}{$\begin{array}{c}\text { Does not } \\
\text { apply }\end{array}$} & \multicolumn{2}{|c|}{$\begin{array}{l}\text { Sometim } \\
\text { es apply }\end{array}$} & \multicolumn{2}{|c|}{$\begin{array}{l}\text { Apply } \\
\text { always }\end{array}$} \\
\hline Results & & $\%$ & & & $\mathrm{Nr}$. & \\
\hline observations & 0 & 00.0 & 1 & 11.11 & 8 & 88.89 \\
\hline self-assessment & 0 & 00.0 & 0 & 00.00 & 10 & 100.0 \\
\hline
\end{tabular}


Table 2. Principle 2: Relationships are deepened by interactions that create a strong attachment.

\begin{tabular}{|c|c|c|c|c|c|c|}
\hline $\begin{array}{c}\text { Indicator } \\
2.1 .\end{array}$ & \multicolumn{6}{|c|}{$\begin{array}{l}\text { Close proximity to the child is } \\
\text { maintained. }\end{array}$} \\
\hline Statements & \multicolumn{2}{|c|}{$\begin{array}{l}\text { Does not } \\
\text { apply }\end{array}$} & \multicolumn{2}{|c|}{$\begin{array}{l}\text { Sometim } \\
\text { es apply }\end{array}$} & \multicolumn{2}{|c|}{$\begin{array}{l}\text { Apply } \\
\text { always }\end{array}$} \\
\hline observations & 0 & & 1 & & 8 & \\
\hline gelf-a & 0 & 000 & 1 & 1000 & 0 & 9000 \\
\hline $\begin{array}{l}\text { Indicator } \\
2.2 \text {. }\end{array}$ & \multicolumn{6}{|c|}{$\begin{array}{l}\text { Attention is drawn to the child's } \\
\text { attempts to communicate (verbal } \\
\text { and non-verbal). }\end{array}$} \\
\hline Statements & \multicolumn{2}{|c|}{$\begin{array}{l}\text { Does not } \\
\text { apply }\end{array}$} & \multicolumn{2}{|c|}{$\begin{array}{l}\text { Sometim } \\
\text { es apply }\end{array}$} & \multicolumn{2}{|c|}{$\begin{array}{l}\text { Apply } \\
\text { always }\end{array}$} \\
\hline Results & $\mathrm{N}$ & $\%$ & & & & \\
\hline observations & 0 & 00.0 & 5 & 55.56 & 4 & 44.44 \\
\hline self-assesgment & 2 & 20.0 & 1 & 10.00 & 7 & 70.00 \\
\hline $\begin{array}{l}\text { Indicator } \\
2.3 \text {. }\end{array}$ & \multicolumn{6}{|c|}{$\begin{array}{l}\text { It reacts quickly to signs of stress in } \\
\text { a soothing and child-friendly way. }\end{array}$} \\
\hline Statements & \multirow{2}{*}{\multicolumn{2}{|c|}{$\begin{array}{l}\text { Does not } \\
\text { apply } \\
\text { Nr. } \%\end{array}$}} & \multirow{2}{*}{\multicolumn{2}{|c|}{$\begin{array}{l}\text { Sometim } \\
\text { es apply } \\
\text { Nr. } \%\end{array}$}} & \multirow{2}{*}{\multicolumn{2}{|c|}{$\begin{array}{r}\text { Apply } \\
\text { always } \\
\text { Nr. } \% \\
\end{array}$}} \\
\hline Results & & & & & & \\
\hline observations & 0 & 00.0 & 0 & 00.00 & 9 & 100.00 \\
\hline self-assessment & 0 & 00.0 & 1 & 10.00 & 9 & 90.00 \\
\hline $\begin{array}{l}\text { Indicator } \\
2.4 \text {. }\end{array}$ & \multicolumn{6}{|c|}{$\begin{array}{l}\text { Children's feelings are respected } \\
\text { including during changes. }\end{array}$} \\
\hline Statements & \multirow{2}{*}{\multicolumn{2}{|c|}{$\begin{array}{l}\text { Does not } \\
\text { apply } \\
\text { Nr. } \%\end{array}$}} & \multirow{2}{*}{\multicolumn{2}{|c|}{$\begin{array}{l}\text { Sometim } \\
\text { es apply } \\
\text { Nr. } \%\end{array}$}} & \multirow{2}{*}{\multicolumn{2}{|c|}{\begin{tabular}{|c|}
\multicolumn{2}{|c|}{ Apply } \\
always \\
$\mathrm{Nr} \quad \%$ \\
\end{tabular}}} \\
\hline Results & & & & & & \\
\hline observations & 0 & 00.0 & 0 & 00.00 & 9 & 100.00 \\
\hline self-assesgment & 0 & 00.0 & 2 & 20.00 & 8 & 80.00 \\
\hline $\begin{array}{l}\text { Indicator } \\
2.5 \text {. }\end{array}$ & \multicolumn{6}{|c|}{$\begin{array}{l}\text { Reaction is consistent } \\
\text { predictable to the child. }\end{array}$} \\
\hline Statements & \multicolumn{2}{|c|}{$\begin{array}{l}\text { Does not } \\
\text { apply }\end{array}$} & \multicolumn{2}{|c|}{$\begin{array}{l}\text { Sometim } \\
\text { es apply }\end{array}$} & \multicolumn{2}{|c|}{$\begin{array}{l}\text { Apply } \\
\text { always }\end{array}$} \\
\hline Results & $\mathrm{N}$ & $\%$ & $\mathrm{~N}$ & & $\mathrm{Nr}$ & \\
\hline observations & 0 & 00.0 & 6 & 66.67 & 3 & 33.33 \\
\hline self-assessment & 0 & 00.0 & 4 & 40.00 & 6 & 60.00 \\
\hline $\begin{array}{l}\text { Indicator } \\
2.6\end{array}$ & \multicolumn{6}{|c|}{$\begin{array}{l}\text { Helpfull to the child to anticipate } \\
\text { the consequences. }\end{array}$} \\
\hline Statements & \multicolumn{2}{|c|}{$\begin{array}{l}\text { Does not } \\
\text { apply }\end{array}$} & \multirow{2}{*}{\multicolumn{2}{|c|}{$\begin{array}{l}\text { Sometim } \\
\text { es apply } \\
\text { Nr. } \%\end{array}$}} & \multirow{2}{*}{\multicolumn{2}{|c|}{$\begin{array}{r}\text { Apply } \\
\text { always } \\
\text { Nr. } \%\end{array}$}} \\
\hline Results & $\mathrm{N}$ & $\%$ & & & & \\
\hline observations & 5 & 55.5 & 4 & 44.44 & 0 & 00.00 \\
\hline self-assessment & 0 & 00.0 & 7 & 70.00 & 3 & 30.00 \\
\hline
\end{tabular}

Table 3. Principle 3: Relationships are promoted through strategies that promote dialogue.

\begin{tabular}{|c|c|c|c|c|c|c|}
\hline Indicator 3.1 . & \multicolumn{6}{|c|}{$\begin{array}{l}\text { Visual contact with the child is } \\
\text { maintained during communication } \\
\text { (where it is culturally accepted). }\end{array}$} \\
\hline Statements & \multirow{2}{*}{\multicolumn{2}{|c|}{$\begin{array}{l}\text { Does not } \\
\text { apply } \\
\mathrm{Nr} . \%\end{array}$}} & \multirow{2}{*}{\multicolumn{2}{|c|}{$\begin{array}{l}\text { Sometim } \\
\text { es apply } \\
\text { Nr. } \%\end{array}$}} & \multirow{2}{*}{\multicolumn{2}{|c|}{$\begin{array}{l}\text { Apply } \\
\text { always } \\
\text { Nr. } \%\end{array}$}} \\
\hline Results & & & & & & \\
\hline obgervations & 0 & 00.0 & 5 & 55.56 & 4 & 44.44 \\
\hline self-assessment & & 00.0 & 0 & 00.00 & 10 & 100.00 \\
\hline Indicator 3.2 . & \multicolumn{6}{|c|}{$\begin{array}{l}\text { The response is warm and loving } \\
\text { towards the child. }\end{array}$} \\
\hline Statements & \multicolumn{2}{|c|}{$\begin{array}{c}\text { Does not } \\
\text { apply }\end{array}$} & \multirow{2}{*}{\multicolumn{2}{|c|}{$\begin{array}{l}\text { Sometim } \\
\text { es apply } \\
\mathrm{Nr} \%\end{array}$}} & \multicolumn{2}{|c|}{$\begin{array}{l}\text { Apply } \\
\text { always }\end{array}$} \\
\hline Results & \multicolumn{2}{|c|}{ Nr. $\%$} & & & \multicolumn{2}{|c|}{ Nr. $\%$} \\
\hline observations & 0 & 00.0 & 0 & 00.00 & 9 & 100.00 \\
\hline self-assessment & 0 & 00.0 & 0 & 00.00 & 10 & 100.00 \\
\hline Indicator 3.3 . & \multicolumn{6}{|c|}{$\begin{array}{l}\text { A calm and attentive expression is } \\
\text { preserved while communicating } \\
\text { with the child. }\end{array}$} \\
\hline Statements & \multirow{2}{*}{\multicolumn{2}{|c|}{$\begin{array}{l}\text { Does not } \\
\text { apply } \\
\text { Nr. } \% \\
\end{array}$}} & \multirow{2}{*}{\multicolumn{2}{|c|}{$\begin{array}{l}\text { Sometim } \\
\text { es apply } \\
\text { Nr. } \% \\
\end{array}$}} & \multirow{2}{*}{\multicolumn{2}{|c|}{$\begin{array}{c}\text { Apply } \\
\text { always } \\
\text { Nr. } \%\end{array}$}} \\
\hline Results & & & & & & \\
\hline observations & 0 & 00.0 & 5 & 55.56 & 4 & 44.44 \\
\hline self-assessment & 0 & 00.0 & 0 & 00.00 & 10 & 100.00 \\
\hline Indicator 3.4 . & \multicolumn{6}{|c|}{$\begin{array}{l}\text { A tone of voice is used that } \\
\text { demonstrates interest, tenderness, } \\
\text { concerm and understanding of the } \\
\text { child. }\end{array}$} \\
\hline Statements & \multirow{2}{*}{\multicolumn{2}{|c|}{$\begin{array}{l}\text { Does not } \\
\text { apply } \\
\text { Nr. } \%\end{array}$}} & \multirow{2}{*}{\multicolumn{2}{|c|}{$\begin{array}{l}\text { Sometim } \\
\text { es apply } \\
\mathrm{Nr} \% \\
\end{array}$}} & \multirow{2}{*}{\multicolumn{2}{|c|}{$\begin{array}{r}\text { Apply } \\
\text { always } \\
\mathrm{Nr} \quad \% \\
\end{array}$}} \\
\hline Results & & & & & & \\
\hline observations & 0 & 00.0 & 0 & 00.00 & 9 & 100.00 \\
\hline self- $\mathrm{a}$ & 0 & 00.0 & 2 & 20.00 & 8 & 80.00 \\
\hline Indicator 3.5 . & \multicolumn{6}{|c|}{$\begin{array}{l}\text { Body language is used, which is } \\
\text { calm, open and expresses interest in } \\
\text { the child. }\end{array}$} \\
\hline Statements & \multicolumn{2}{|c|}{$\begin{array}{c}\text { Does not } \\
\text { apply }\end{array}$} & \multicolumn{2}{|c|}{$\begin{array}{l}\text { Sometim } \\
\text { es apply }\end{array}$} & \multicolumn{2}{|c|}{$\begin{array}{c}\text { Apply } \\
\text { always }\end{array}$} \\
\hline Results & & $\%$ & & & $\mathrm{Nr}$. & \\
\hline observations & 0 & 00.0 & 0 & 00.00 & 9 & 100.00 \\
\hline self-asses & 0 & 00.0 & 3 & 33.33 & 7 & 70.00 \\
\hline
\end{tabular}


Table 4. Principle 4: Relationships between the children themselves are stimulated.

\begin{tabular}{|c|c|c|c|c|c|c|}
\hline $\begin{array}{c}\text { Indicator } \\
4.1 .\end{array}$ & \multicolumn{6}{|c|}{$\begin{array}{l}\text { The importance of peer } \\
\text { relationships in very young children } \\
\text { is recognized. }\end{array}$} \\
\hline Statements & \multirow{2}{*}{\multicolumn{2}{|c|}{$\begin{array}{l}\text { Does not } \\
\text { apply } \\
\text { Nr. } \%\end{array}$}} & \multirow{2}{*}{\multicolumn{2}{|c|}{$\begin{array}{l}\text { Sometim } \\
\text { es apply } \\
\text { Nr. } \%\end{array}$}} & \multicolumn{2}{|c|}{$\begin{array}{l}\text { Apply } \\
\text { always }\end{array}$} \\
\hline Results & & & & & & \\
\hline observations & 0 & 00.0 & 9 & 100.0 & 0 & 00.00 \\
\hline gelf-assessment & 0 & 00.0 & 1 & 10.00 & 9 & 90.00 \\
\hline $\begin{array}{c}\text { Indicator } \\
4.2 \text {. }\end{array}$ & \multicolumn{6}{|c|}{$\begin{array}{l}\text { Social interactions between children } \\
\text { are encouraged through modeling, } \\
\text { careful guidance and, where } \\
\text { necessary, intervention. }\end{array}$} \\
\hline Statements & \multirow{2}{*}{\multicolumn{2}{|c|}{$\begin{array}{l}\text { Does not } \\
\text { apply } \\
\text { Nr. } \%\end{array}$}} & \multicolumn{2}{|c|}{$\begin{array}{l}\text { Sometim } \\
\text { es apply }\end{array}$} & \multicolumn{2}{|c|}{$\begin{array}{c}\text { Apply } \\
\text { always }\end{array}$} \\
\hline Results & & & & & & \\
\hline observations & 0 & 00.0 & 8 & 77.78 & 1 & 11.11 \\
\hline gelf-assesgn & 0 & 00.0 & 1 & 10.00 & 9 & 90.00 \\
\hline $\begin{array}{c}\text { Indicator } \\
4.3\end{array}$ & \multicolumn{6}{|c|}{$\begin{array}{l}\text { The child is encouraged to express } \\
\text { their emotions appropriately using a } \\
\text { language that is available to them. }\end{array}$} \\
\hline Statements & \multirow{2}{*}{\multicolumn{2}{|c|}{$\begin{array}{l}\text { Does not } \\
\text { apply } \\
\text { Nr. } \%\end{array}$}} & \multirow{2}{*}{\multicolumn{2}{|c|}{$\begin{array}{l}\text { Sometim } \\
\text { es apply } \\
\text { Nr. } \%\end{array}$}} & \multirow{2}{*}{\multicolumn{2}{|c|}{$\begin{array}{r}\text { Apply } \\
\text { always } \\
\text { Nr. } \quad \% \\
\end{array}$}} \\
\hline Results & & & & & & \\
\hline observations & 0 & 00.0 & 4 & 44.44 & 5 & 55.56 \\
\hline gelf-asseasment & 0 & 00.0 & 3 & 30.00 & 7 & 70.00 \\
\hline $\begin{array}{c}\text { Indicator } \\
4.4 .\end{array}$ & \multicolumn{6}{|c|}{$\begin{array}{l}\text { Helping very young children to } \\
\text { understand that others also have } \\
\text { needs and feelings, and that they } \\
\text { need to be taken into account. }\end{array}$} \\
\hline Statements & \multirow{2}{*}{\multicolumn{2}{|c|}{$\begin{array}{l}\text { Does not } \\
\text { apply } \\
\mathrm{Nr} . \%\end{array}$}} & \multicolumn{2}{|c|}{$\begin{array}{l}\text { Sometim } \\
\text { es apply }\end{array}$} & \multicolumn{2}{|c|}{$\begin{array}{c}\text { Apply } \\
\text { always }\end{array}$} \\
\hline Results & & & & & $\mathrm{N}$ & \\
\hline observations & 0 & 00.0 & 9 & 100.0 & 0 & 00.0 \\
\hline self-assessment & 0 & 00.0 & 1 & 10.00 & 9 & 90.00 \\
\hline
\end{tabular}

Table 5. Principle 5: Relationships support / facilitate children under the age of three in changing their day-to-day and extraordinary activities.

\begin{tabular}{|c|c|c|c|c|c|c|}
\hline $\begin{array}{c}\text { Indicator } \\
5.1 .\end{array}$ & \multicolumn{6}{|c|}{$\begin{array}{l}\text { The individual nature of each child's } \\
\text { approach to change is recognized } \\
\text { and supports these changes. }\end{array}$} \\
\hline Statements & \multirow{2}{*}{\multicolumn{2}{|c|}{$\begin{array}{l}\text { Does not } \\
\text { apply } \\
\text { Nr. } \%\end{array}$}} & \multirow{2}{*}{\multicolumn{2}{|c|}{$\begin{array}{l}\text { Sometim } \\
\text { es apply } \\
\text { Nr. } \%\end{array}$}} & \multirow{2}{*}{\multicolumn{2}{|c|}{$\begin{array}{r}\text { Apply } \\
\text { always } \\
\text { Nr. } \% \\
\end{array}$}} \\
\hline Results & & & & & & \\
\hline observations & 0 & 00.0 & 8 & 88.89 & 1 & 11.11 \\
\hline self-assesgment & 0 & 00.0 & 8 & 88.89 & 1 & 11.11 \\
\hline $\begin{array}{c}\text { Indicator } \\
5.2 \text {. }\end{array}$ & \multicolumn{6}{|c|}{$\begin{array}{l}\text { Communication and coordination } \\
\text { with all other adults who are } \\
\text { involved with the child at the time } \\
\text { of change is maintained. }\end{array}$} \\
\hline Statements & \multicolumn{2}{|c|}{$\begin{array}{l}\text { Does not } \\
\text { apply } \\
\text { Nr. } \%\end{array}$} & \multicolumn{2}{|c|}{$\begin{array}{l}\text { Sometim } \\
\text { es apply }\end{array}$} & \multicolumn{2}{|c|}{$\begin{array}{l}\text { Apply } \\
\text { always }\end{array}$} \\
\hline observations & . & & $N_{-1}$ & & & \\
\hline & & 00.0 & 1 & & & 00.03 \\
\hline & 0 & 00.0 & 1 & 10.00 & 9 & 90.00 \\
\hline $\begin{array}{c}\text { Indicator } \\
5.3 .\end{array}$ & \multicolumn{6}{|c|}{$\begin{array}{l}\text { Daily activities are regularly } \\
\text { evaluated to ensure that the child's } \\
\text { needs are met by observing the } \\
\text { child's reactions. }\end{array}$} \\
\hline Statements & \multicolumn{2}{|c|}{$\begin{array}{l}\text { Does not } \\
\text { apply } \\
\text { Nr. } \%\end{array}$} & \multicolumn{2}{|c|}{$\begin{array}{l}\text { Sometim } \\
\text { es apply } \\
\text { Nr. } \%\end{array}$} & \multicolumn{2}{|c|}{$\begin{array}{c}\text { Apply } \\
\text { always } \\
\text { Nr. } \%\end{array}$} \\
\hline observations & 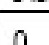 & & 4 & & & . \\
\hline gelf-assessment & 1 & 10.0 & 2 & 20.00 & 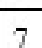 & 70.00 \\
\hline $\begin{array}{c}\text { Indicator } \\
5.4 .\end{array}$ & \multicolumn{6}{|c|}{$\begin{array}{l}\text { Regular communication with } \\
\text { parents / families about activities to } \\
\text { ensure their consistency, is main- } \\
\text { tained. }\end{array}$} \\
\hline Statements & \multicolumn{2}{|c|}{$\begin{array}{l}\text { Does not } \\
\text { apply } \\
\mathrm{Nr} \quad \%\end{array}$} & \multicolumn{2}{|c|}{$\begin{array}{l}\text { Sometime } \\
\text { s apply } \\
\mathrm{Nr} \quad \% \\
\end{array}$} & \multicolumn{2}{|c|}{$\begin{array}{r}\text { Apply } \\
\text { always } \\
\mathrm{Nr} \quad \%\end{array}$} \\
\hline observations & 0 & 00.0 & 5 & 55.56 & 4 & 44.44 \\
\hline self-a & 1 & 10.0 & 1 & 10.00 & 8 & 88.89 \\
\hline
\end{tabular}




\subsection{Priority area "Family and Community"}

Table 6. Principle 1: Knowing and respecting families and communities affirms their relationship with them and between them.

\begin{tabular}{|c|c|c|c|c|c|c|}
\hline $\begin{array}{c}\text { Indicator } \\
1.1 .\end{array}$ & \multicolumn{6}{|c|}{$\begin{array}{l}\text { The specialist studies the values, } \\
\text { beliefs, assumptions and practices of } \\
\text { the family and applies them } \\
\text { whenever possible. }\end{array}$} \\
\hline $\begin{array}{l}\text { Statements } \\
\text { Results }\end{array}$ & \multicolumn{2}{|c|}{$\begin{array}{l}\text { Does not } \\
\text { apply } \\
\mathrm{Nr} \quad \%\end{array}$} & \multicolumn{2}{|c|}{$\begin{array}{l}\text { Sometim } \\
\text { es apply } \\
\mathrm{Nr} \quad \%\end{array}$} & \multicolumn{2}{|c|}{$\begin{array}{c}\text { Apply } \\
\text { always } \\
\mathrm{Nr} \quad \% \\
\end{array}$} \\
\hline observations & 0 & 00.0 & 9 & 100.0 & 0 & 00.00 \\
\hline self-asseasment & 0 & 00.0 & 8 & 80.00 & 2 & 20.00 \\
\hline $\begin{array}{c}\text { Indicator } \\
1.2 .\end{array}$ & \multicolumn{6}{|c|}{$\begin{array}{l}\text { The specialist studies the } \\
\text { communities in which children live } \\
\text { and applies this lonowledge to meet } \\
\text { the needs of children to create } \\
\text { learning approaches. }\end{array}$} \\
\hline $\begin{array}{l}\text { Statements } \\
\text { Results }\end{array}$ & \multicolumn{2}{|c|}{$\begin{array}{l}\text { Does not } \\
\text { apply } \\
\mathrm{Nr} \quad \%\end{array}$} & \multicolumn{2}{|c|}{$\begin{array}{l}\text { Sometim } \\
\text { es apply } \\
\mathrm{Nr} \quad \%\end{array}$} & \multicolumn{2}{|c|}{$\begin{array}{c}\text { Apply } \\
\text { always } \\
\mathrm{Nr} \quad \% \\
\end{array}$} \\
\hline observations & 0 & 00.0 & 4 & 44.44 & 5 & 55.56 \\
\hline self-asseasment & 1 & 10.0 & 7 & 70.00 & 2 & 20.00 \\
\hline $\begin{array}{c}\text { Indicator } \\
1.3 .\end{array}$ & \multicolumn{6}{|c|}{$\begin{array}{l}\text { The specialist builds on the } \\
\text { strengths of the family and } \\
\text { community, and when it is possible } \\
\text { to adopt and incorporate } \\
\text { "lenowledge funds" that are part of } \\
\text { every family and community. }\end{array}$} \\
\hline Statements & \multicolumn{2}{|c|}{$\begin{array}{l}\text { Does not } \\
\text { apply } \\
\mathrm{Nr} \quad \%\end{array}$} & \multicolumn{2}{|c|}{$\begin{array}{r}\text { Sometim } \\
\text { es apply } \\
\mathrm{Nr} \quad \% \\
\end{array}$} & \multicolumn{2}{|c|}{$\begin{array}{c}\text { Apply } \\
\text { always } \\
\mathrm{Nr} \quad \% \\
\end{array}$} \\
\hline observations & 0 & 00.0 & 8 & 88.89 & 1 & 11.11 \\
\hline self-asseasment & 2 & 20.0 & 4 & 40.00 & 4 & 40.00 \\
\hline $\begin{array}{c}\text { Indicator } \\
1.4 .\end{array}$ & \multicolumn{6}{|c|}{$\begin{array}{l}\text { The specialist personalizes } \\
\text { relationships and services for } \\
\text { families in the way they best meet } \\
\text { their needs. }\end{array}$} \\
\hline Statements & \multicolumn{2}{|c|}{$\begin{array}{l}\text { Does not } \\
\text { apply } \\
\mathrm{Nr} \%\end{array}$} & \multicolumn{2}{|c|}{\begin{tabular}{r|} 
Sometim \\
es apply \\
$\mathrm{Nr} \quad \%$ \\
\end{tabular}} & \multicolumn{2}{|c|}{$\begin{array}{c}\text { Apply } \\
\text { always } \\
\mathrm{Nr} \quad \% \\
\end{array}$} \\
\hline observations & 0 & 00.0 & 3 & 33.33 & 6 & 66.67 \\
\hline gelf-zasessment & 0 & 00.0 & 1 & 10.00 & 9 & 90.00 \\
\hline $\begin{array}{c}\text { Indicator } \\
1.5 .\end{array}$ & \multicolumn{6}{|c|}{$\begin{array}{l}\text { The specialist encourages families } \\
\text { to leam from one another and to } \\
\text { support them. }\end{array}$} \\
\hline Statements & \multicolumn{2}{|c|}{$\begin{array}{l}\text { Does not } \\
\text { apply } \\
\mathrm{Nr} \quad \%\end{array}$} & \multicolumn{2}{|c|}{$\begin{array}{r}\text { Sometim } \\
\text { es apply } \\
\mathrm{Nr} \quad \% \\
\end{array}$} & \multicolumn{2}{|c|}{\begin{tabular}{|c|c|}
\multicolumn{2}{|c|}{ Apply } \\
always \\
$\mathrm{Nr} \quad \%$ \\
\end{tabular}} \\
\hline vations & 0 & 00.0 & 7 & 77.78 & 2 & 22.22 \\
\hline self-assessment & 1 & 10.0 & 3 & 30.00 & 6 & 60.00 \\
\hline
\end{tabular}

Table 7. Principle 2: Sensitive, respectful and equal communication with families supports the development and learning of children.

\begin{tabular}{|c|c|c|c|c|c|c|}
\hline $\begin{array}{c}\text { Indicator } \\
2.1\end{array}$ & \multicolumn{6}{|c|}{$\begin{array}{l}\text { The specialist engages in } \\
\text { continuous, } \\
\text { communication with parents } \\
\text { families to share information about } \\
\text { the child's experiences, health and } \\
\text { needs. }\end{array}$} \\
\hline Statements & \multirow{2}{*}{\multicolumn{2}{|c|}{$\begin{array}{l}\text { Does not } \\
\text { apply } \\
\mathrm{Nr} . \%\end{array}$}} & \multicolumn{2}{|c|}{$\begin{array}{l}\text { Sometim } \\
\text { es apply }\end{array}$} & \multicolumn{2}{|c|}{$\begin{array}{l}\text { Apply } \\
\text { always }\end{array}$} \\
\hline Results & & & \multicolumn{2}{|c|}{ Nr. $\%$} & \multicolumn{2}{|c|}{ Nr. $\quad \%$} \\
\hline observations & 0 & 00.0 & 0 & 00.00 & 9 & 100.00 \\
\hline self-assessment & 0 & 00.0 & 1 & 10.00 & 9 & 90.00 \\
\hline $\begin{array}{c}\text { Indicator } \\
2.2 .\end{array}$ & \multicolumn{6}{|c|}{$\begin{array}{l}\text { The specialist spends time listening } \\
\text { carefully by being unbiased to the } \\
\text { families. }\end{array}$} \\
\hline Statements & \multicolumn{2}{|c|}{$\begin{array}{l}\text { Does not } \\
\text { apply }\end{array}$} & \multicolumn{2}{|c|}{$\begin{array}{l}\text { Sometim } \\
\text { es apply }\end{array}$} & \multicolumn{2}{|c|}{$\begin{array}{l}\text { Apply } \\
\text { always }\end{array}$} \\
\hline Results & \multicolumn{2}{|c|}{$\mathrm{Nr} . \quad \%$} & \multicolumn{2}{|c|}{ Nr. $\%$} & \multicolumn{2}{|c|}{ Nr. $\quad \%$} \\
\hline observations & 0 & 00.0 & 6 & 66.67 & 3 & 33.33 \\
\hline self-assessment & 0 & 00.0 & 2 & 20.00 & 8 & 80.00 \\
\hline $\begin{array}{c}\text { Indicator } \\
2.3\end{array}$ & \multicolumn{6}{|c|}{$\begin{array}{l}\text { The specialist uses different forms } \\
\text { of communication with families, } \\
\text { including their language and } \\
\text { communication preferences. }\end{array}$} \\
\hline Statements & \multicolumn{2}{|c|}{$\begin{array}{l}\text { Does not } \\
\text { apply }\end{array}$} & \multicolumn{2}{|c|}{$\begin{array}{l}\text { Sometim } \\
\text { es apply }\end{array}$} & \multicolumn{2}{|c|}{$\begin{array}{l}\text { Apply } \\
\text { always }\end{array}$} \\
\hline Results & \multicolumn{2}{|c|}{$\mathrm{Nr} . \quad \%$} & \multicolumn{2}{|c|}{ Nr. $\%$} & \multicolumn{2}{|c|}{ Nr. $\quad \%$} \\
\hline observations & 0 & 00.0 & 4 & 44.44 & 5 & 55.56 \\
\hline self-assessment & 0 & 00.0 & 6 & 60.00 & 4 & 40.00 \\
\hline $\begin{array}{c}\text { Indicator } \\
2.4\end{array}$ & \multicolumn{6}{|c|}{$\begin{array}{l}\text { The specialist maintains the } \\
\text { confidentiality of all information } \\
\text { about the child and his / her family. }\end{array}$} \\
\hline Statements & \multicolumn{2}{|c|}{$\begin{array}{c}\text { Does not } \\
\text { apply }\end{array}$} & \multicolumn{2}{|c|}{$\begin{array}{l}\text { Sometim } \\
\text { es apply }\end{array}$} & \multicolumn{2}{|c|}{$\begin{array}{l}\text { Apply } \\
\text { always }\end{array}$} \\
\hline Results & \multicolumn{2}{|c|}{ Nr. $\%$} & \multicolumn{2}{|c|}{ Nr. $\%$} & \multicolumn{2}{|c|}{ Nr. $\%$} \\
\hline observations & 0 & 00.0 & 1 & 11.11 & 8 & 88.89 \\
\hline self-assessment & 0 & 00.0 & 1 & 10.00 & 9 & 90.00 \\
\hline $\begin{array}{c}\text { Indicator } \\
2.5 \text {. }\end{array}$ & \multicolumn{6}{|c|}{$\begin{array}{l}\text { The specialist moderates } \\
\text { disagreements, reports and supports } \\
\text { in ways that lead to positive results } \\
\text { for the child. }\end{array}$} \\
\hline Statements & \multicolumn{2}{|c|}{$\begin{array}{c}\text { Does not } \\
\text { apply }\end{array}$} & \multicolumn{2}{|c|}{$\begin{array}{l}\text { Sometim } \\
\text { es apply }\end{array}$} & \multicolumn{2}{|c|}{$\begin{array}{l}\text { Apply } \\
\text { always }\end{array}$} \\
\hline Results & $\mathrm{Nr}$ & $\%$ & & $\%$ & $\mathrm{Nr}$ & $\%$ \\
\hline observations & 0 & 00.0 & 4 & 44.44 & 5 & 55.56 \\
\hline self-assessment & 0 & 00.0 & 4 & 40.00 & 6 & 60.00 \\
\hline $\begin{array}{c}\text { Indicator } \\
2.6\end{array}$ & & $\begin{array}{l}\text { speci } \\
\text { effec } \\
\text { ats } \\
\text { matic } \\
\text { rienc }\end{array}$ & & $\begin{array}{l}\text { ngages } \\
\text { commu } \\
\text { amilies } \\
\text { about } \\
\text { alth an }\end{array}$ & & $\begin{array}{l}\text { tinuous } \\
\text { share } \\
\text { child's }\end{array}$ \\
\hline Statements & & $\begin{array}{l}\text { s not } \\
\text { ply }\end{array}$ & & $\begin{array}{l}\text { metim } \\
\text { apply }\end{array}$ & & $\begin{array}{l}\text { pply } \\
\text { ways }\end{array}$ \\
\hline Results & $\mathrm{Nr}$ & $\%$ & & & $\mathrm{Nr}$ & $\%$ \\
\hline observations & 2 & 22.2 & 4 & 44.44 & 3 & 33.3 \\
\hline self-assessment & 0 & 00.0 & 1 & 10.00 & 9 & 90.00 \\
\hline
\end{tabular}


Table 8. Principle 3: Services are best provided in partnership with the family.

\begin{tabular}{|c|c|c|c|c|c|c|}
\hline $\begin{array}{c}\text { Indicator } \\
3.1 \text {. }\end{array}$ & \multicolumn{6}{|c|}{$\begin{array}{l}\text { The right and responsibility of each } \\
\text { family to be involved in making the } \\
\text { final decisions about the } \\
\text { development, learning, well-being } \\
\text { and services provided to the child is } \\
\text { guaranteed. }\end{array}$} \\
\hline Statements & \multirow{2}{*}{\multicolumn{2}{|c|}{$\begin{array}{l}\text { Does not } \\
\text { apply } \\
\text { Nr. } \%\end{array}$}} & \multirow{2}{*}{\multicolumn{2}{|c|}{$\begin{array}{l}\text { Sometim } \\
\text { es apply } \\
\text { Nr. } \%\end{array}$}} & \multirow{2}{*}{\multicolumn{2}{|c|}{$\begin{array}{c}\text { Apply } \\
\text { always } \\
\text { Nr. } \%\end{array}$}} \\
\hline Results & & & & & & \\
\hline observations & 0 & 00.0 & 4 & 44.44 & 5 & 55.56 \\
\hline gelf-assessment & 0 & 00.0 & 1 & 10.00 & 9 & 90.00 \\
\hline $\begin{array}{c}\text { Indicator } \\
3.2 \text {. }\end{array}$ & \multicolumn{6}{|c|}{$\begin{array}{l}\text { Family-specific goals, needs and } \\
\text { cultural practices are respected and } \\
\text { mutually agree activities to enhance } \\
\text { continuity between home and other } \\
\text { backgrounds. }\end{array}$} \\
\hline Statements & \multicolumn{2}{|c|}{$\begin{array}{l}\text { Does not } \\
\text { apply } \\
\text { Nr. } \%\end{array}$} & \multicolumn{2}{|c|}{$\begin{array}{l}\text { Sometim } \\
\text { es apply } \\
\text { Nr. } \%\end{array}$} & \multicolumn{2}{|c|}{$\begin{array}{c}\text { Apply } \\
\text { always } \\
\text { Nr. } \%\end{array}$} \\
\hline observations & 1 & 11.1 & 3 & 33.33 & 5 & 55.56 \\
\hline self-assessment & 0 & 00.0 & 4 & 40.00 & 6 & 60.00 \\
\hline $\begin{array}{c}\text { Indicator } \\
3.3 \text {. }\end{array}$ & \multicolumn{6}{|c|}{$\begin{array}{l}\text { Strengthening and strengthening } \\
\text { parenting practices while offering } \\
\text { proven parental education and } \\
\text { support when needed. }\end{array}$} \\
\hline Statements & \multicolumn{2}{|c|}{$\begin{array}{l}\text { Does not } \\
\text { apply } \\
\text { Nr. } \%\end{array}$} & \multicolumn{2}{|c|}{$\begin{array}{l}\text { Sometim } \\
\text { es apply } \\
\text { Nr. } \%\end{array}$} & \multicolumn{2}{|c|}{$\begin{array}{c}\text { Apply } \\
\text { always } \\
\text { Nr. } \%\end{array}$} \\
\hline observations & 6 & 66.6 & 3 & 33.33 & 0 & 00.00 \\
\hline gelf-assessment & 2 & 20.0 & 6 & 60.00 & 2 & 20.00 \\
\hline $\begin{array}{c}\text { Indicator } \\
3.4 .\end{array}$ & \multicolumn{6}{|c|}{$\begin{array}{l}\text { The involvement and commitment } \\
\text { of fathers and other family members } \\
\text { to the care and learning of the child } \\
\text { is encouraged. }\end{array}$} \\
\hline Statements & \multicolumn{2}{|c|}{$\begin{array}{l}\text { Does not } \\
\text { apply } \\
\text { Nr. } \%\end{array}$} & \multicolumn{2}{|c|}{$\begin{array}{l}\text { Sometim } \\
\text { es apply } \\
\text { Nr. } \%\end{array}$} & \multicolumn{2}{|c|}{$\begin{array}{r}\text { Apply } \\
\text { always } \\
\text { Nr. } \quad \% \\
\end{array}$} \\
\hline observations & 1 & 11.1 & 8 & 88.89 & 0 & 00.00 \\
\hline gelf-assessment & 0 & 00.0 & 1 & 10.00 & 9 & 90.00 \\
\hline
\end{tabular}

Table 9. Principle 4: Partnerships based on co-operative and community-based cooperation best support families and children.

\begin{tabular}{|c|c|c|c|c|c|c|}
\hline $\begin{array}{c}\text { Indicator } \\
4.1 .\end{array}$ & \multicolumn{6}{|c|}{$\begin{array}{l}\text { The specialist guarantees } \\
\text { trouble-free transitions by } \\
\text { negotiating with various services to } \\
\text { support families. }\end{array}$} \\
\hline Statements & \multicolumn{2}{|c|}{$\begin{array}{l}\text { Does not } \\
\text { apply } \\
\text { Nr. } \%\end{array}$} & \multicolumn{2}{|c|}{$\begin{array}{l}\text { Sometim } \\
\text { es apply } \\
\text { Nr. } \%\end{array}$} & \multicolumn{2}{|c|}{$\begin{array}{c}\text { Apply } \\
\text { always } \\
\text { Nr. } \%\end{array}$} \\
\hline observations & 1 & 11.1 & 3 & 33.33 & 6 & 66.67 \\
\hline gelf-assessment & 9 & 90.0 & 1 & 10.00 & 0 & 00.00 \\
\hline $\begin{array}{c}\text { Indicator } \\
4.2 \text {. }\end{array}$ & \multicolumn{6}{|c|}{$\begin{array}{l}\text { The specialist actively engages local } \\
\text { communities to promote and } \\
\text { promote the rights of children and } \\
\text { families through advocacy activities } \\
\text { that go beyond local communities. }\end{array}$} \\
\hline Statements & \multicolumn{2}{|c|}{$\begin{array}{c}\text { Does not } \\
\text { apply }\end{array}$} & \multicolumn{2}{|c|}{$\begin{array}{l}\text { Sometim } \\
\text { es apply }\end{array}$} & \multicolumn{2}{|c|}{$\begin{array}{l}\text { Apply } \\
\text { always }\end{array}$} \\
\hline Results & \multicolumn{2}{|c|}{ Nr. $\%$} & \multicolumn{2}{|c|}{ Nr. $\%$} & \multicolumn{2}{|c|}{ Nr. $\%$} \\
\hline observations & 1 & 11.1 & 8 & 88.89 & 0 & 00.00 \\
\hline gelf-assesgment & 8 & 80.0 & 0 & 00.00 & 2 & 20.00 \\
\hline $\begin{array}{c}\text { Indicator } \\
4.3 .\end{array}$ & \multicolumn{6}{|c|}{$\begin{array}{l}\text { The specialist creates opportunities } \\
\text { for community members to be } \\
\text { involved and take part in early } \\
\text { childhood service activities. }\end{array}$} \\
\hline Statements & \multicolumn{2}{|c|}{$\begin{array}{l}\text { Does not } \\
\text { apply } \\
\text { Nr. } \%\end{array}$} & \multicolumn{2}{|c|}{$\begin{array}{l}\text { Sometim } \\
\text { es apply } \\
\text { Nr. } \%\end{array}$} & \multicolumn{2}{|c|}{$\begin{array}{c}\text { Apply } \\
\text { always } \\
\text { Nr. } \%\end{array}$} \\
\hline observations & 6 & 66.6 & 3 & 33.33 & 0 & 00.00 \\
\hline gelf-assessment & 8 & 80.0 & 1 & 10.00 & 1 & 10.00 \\
\hline $\begin{array}{c}\text { Indicator } \\
4.4 .\end{array}$ & \multicolumn{6}{|c|}{$\begin{array}{l}\text { The specialist notes that very young } \\
\text { children are part of the community } \\
\text { and play an important role in their } \\
\text { early life experience. }\end{array}$} \\
\hline Statements & \multicolumn{2}{|c|}{$\begin{array}{l}\text { Does not } \\
\text { apply } \\
\text { Nr. } \%\end{array}$} & \multicolumn{2}{|c|}{$\begin{array}{l}\text { Sometim } \\
\text { es apply } \\
\text { Nr. } \%\end{array}$} & \multicolumn{2}{|c|}{$\begin{array}{r}\text { Apply } \\
\text { always } \\
\text { Nr. } \% \\
\end{array}$} \\
\hline observations & 1 & 11.1 & 3 & 33.33 & 5 & 55.56 \\
\hline gelf-assesament & 0 & 00.0 & 4 & 40.00 & 6 & 60.00 \\
\hline
\end{tabular}




\subsection{Priority area "Inclusiveness, diversity and values of democracy"}

Table 10. Principle 1: Inclusion provides equal opportunities for each child and family to participate regardless of gender, race, ethnic origin, culture, mother tongue, religion, family structure, social status, age or special needs.

\begin{tabular}{|c|c|c|c|c|c|c|}
\hline $\begin{array}{l}\text { Indicator } \\
\text { 1.1. }\end{array}$ & \multicolumn{6}{|c|}{$\begin{array}{l}\text { The specialist demonstrates } \\
\text { awareness of the values, culture, } \\
\text { beliefs, assumptions and attitudes } \\
\text { about how they affect } \\
\text { communication, interactions and } \\
\text { relationships between children and } \\
\text { their families. }\end{array}$} \\
\hline $\begin{array}{l}\text { Statements } \\
\text { Results } \\
\end{array}$ & \multicolumn{2}{|c|}{$\begin{array}{l}\text { Does not } \\
\text { apply } \\
\mathrm{Nr} \quad \%\end{array}$} & \multicolumn{2}{|c|}{\begin{tabular}{|l|} 
Sometim \\
es apply \\
$\mathrm{Nr} \quad \%$ \\
\end{tabular}} & \multicolumn{2}{|c|}{$\begin{array}{c}\text { Apply } \\
\text { always } \\
\mathrm{Nr} \quad \% \\
\end{array}$} \\
\hline observations & 2 & 22.2 & 7 & 77.78 & 0 & 00.00 \\
\hline self-asseasment & 1 & 10.0 & 8 & 80.00 & 0 & 00.00 \\
\hline $\begin{array}{c}\text { Indicator } \\
1.2 .\end{array}$ & \multicolumn{6}{|c|}{$\begin{array}{l}\text { The specialist treats each child and } \\
\text { his family with respect and dignity. }\end{array}$} \\
\hline $\begin{array}{l}\text { Statements } \\
\text { Results } \\
\end{array}$ & \multicolumn{2}{|c|}{$\begin{array}{l}\text { Does not } \\
\text { apply } \\
\mathrm{Nr} \quad \%\end{array}$} & \multicolumn{2}{|c|}{ 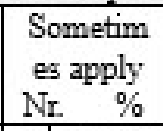 } & \multicolumn{2}{|c|}{$\begin{array}{r}\text { Apply } \\
\text { always } \\
\mathrm{Nr} \quad \% \\
\end{array}$} \\
\hline observations & 2 & 22.2 & 1 & 11.11 & 6 & 66.67 \\
\hline self-assessment & 0 & 00.0 & 1 & 10.00 & 9 & 90.00 \\
\hline $\begin{array}{l}\text { Indicator } \\
1.3 .\end{array}$ & \multicolumn{6}{|c|}{$\begin{array}{l}\text { The specialist adapts the } \\
\text { environment, leaming practices and } \\
\text { interactions so that those with } \\
\text { different physical and mental } \\
\text { abilities or who speak different } \\
\text { languages can also participate fully. }\end{array}$} \\
\hline Statements & \multicolumn{2}{|c|}{$\begin{array}{l}\text { Does not } \\
\text { apply } \\
\mathrm{Nr} \quad \%\end{array}$} & \multicolumn{2}{|c|}{ 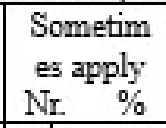 } & \multicolumn{2}{|c|}{$\begin{array}{r}\text { Apply } \\
\text { always } \\
\mathrm{Nr} \quad \% \\
\end{array}$} \\
\hline observations & 2 & 22.2 & 6 & 66.67 & 1 & 11.11 \\
\hline self-assessment & 0 & 00.0 & 2 & 20.00 & 8 & 80.00 \\
\hline $\begin{array}{l}\text { Indicator } \\
1.4 .\end{array}$ & \multicolumn{6}{|c|}{$\begin{array}{l}\text { The specialist draws attention to } \\
\text { gender and other stereotypes } \\
\text { (including stereotypes about } \\
\text { poverty) in the materials and } \\
\text { learning practices provided to } \\
\text { children and/or their families. }\end{array}$} \\
\hline Statements & \multicolumn{2}{|c|}{$\begin{array}{c}\text { Does not } \\
\text { apply } \\
\mathrm{Nr} \quad \%\end{array}$} & \multicolumn{2}{|c|}{$\begin{array}{l}\text { Sometim } \\
\text { es apply } \\
\mathrm{Nr} \quad \%\end{array}$} & \multicolumn{2}{|c|}{$\begin{array}{l}\text { Apply } \\
\text { always }\end{array}$} \\
\hline observations & 3 & 33.3 & 6 & 66.67 & 0 & 00.00 \\
\hline self-zasessment & 3 & 33.3 & 2 & 20.00 & 5 & 50.00 \\
\hline $\begin{array}{l}\text { Indicator } \\
1.5 .\end{array}$ & \multicolumn{6}{|c|}{$\begin{array}{l}\text { The specialist provides a level } \\
\text { playing field for all girls and boys in } \\
\text { the gervices for young children. }\end{array}$} \\
\hline Statements & \multicolumn{2}{|c|}{$\begin{array}{l}\text { Does not } \\
\text { apply } \\
\mathrm{Nr} \quad \%\end{array}$} & \multicolumn{2}{|c|}{$\begin{array}{l}\begin{array}{l}\text { Sometim } \\
\text { es apply } \\
\mathrm{Nr} \quad \%\end{array} \\
\end{array}$} & \multicolumn{2}{|c|}{$\begin{array}{r}\text { Apply } \\
\text { always } \\
\mathrm{Nr} \quad \%\end{array}$} \\
\hline observations & 3 & 33.3 & 0 & 00.00 & 6 & 66.67 \\
\hline self-assessment & 0 & 00.0 & 1 & 10.00 & 10 & 10.00 \\
\hline
\end{tabular}

Table 11. Principle 2: Understanding and appreciating the diversity that exists among children, families and communities builds childhood identities.

\begin{tabular}{|c|c|c|c|c|c|c|}
\hline $\begin{array}{l}\text { Indicator } \\
2.1 \text {. }\end{array}$ & \multicolumn{6}{|c|}{$\begin{array}{l}\text { Includes interactions, learning } \\
\text { practices and materials that reflect } \\
\text { the cultural, linguistic, family and } \\
\text { individual diversity of children and } \\
\text { their families. }\end{array}$} \\
\hline Statements & \multicolumn{2}{|c|}{$\begin{array}{c}\text { Does not } \\
\text { apply }\end{array}$} & \multicolumn{2}{|c|}{$\begin{array}{l}\text { Sometim } \\
\text { es apply }\end{array}$} & \multicolumn{2}{|c|}{$\begin{array}{l}\text { Apply } \\
\text { always }\end{array}$} \\
\hline Results & \multicolumn{2}{|c|}{ Nr. $\%$} & \multicolumn{2}{|c|}{ Nr. $\%$} & $\mathrm{Nr}$. & $\%$ \\
\hline observations & 3 & 33.3 & 5 & 55.56 & 1 & 11.11 \\
\hline self-assessment & 0 & 00.0 & 9 & 90.00 & 1 & 10.00 \\
\hline $\begin{array}{l}\text { Indicator } \\
2.2 \text {. }\end{array}$ & \multicolumn{6}{|c|}{$\begin{array}{l}\text { Helps family upbringing with } \\
\text { children, in addition to cultural and } \\
\text { language styles wherever possible. } \\
\text { Where appropriate, appropriate } \\
\text { support and guidance is offered. }\end{array}$} \\
\hline Statements & \multicolumn{2}{|c|}{$\begin{array}{l}\text { Does not } \\
\text { apply }\end{array}$} & \multicolumn{2}{|c|}{$\begin{array}{l}\text { Sometim } \\
\text { es apply }\end{array}$} & \multicolumn{2}{|c|}{$\begin{array}{l}\text { Apply } \\
\text { always }\end{array}$} \\
\hline Results & \multicolumn{2}{|c|}{ Nr. $\%$} & \multicolumn{2}{|c|}{ Nr. $\%$} & \multicolumn{2}{|c|}{ Nr. $\%$} \\
\hline observations & 2 & 22.2 & 6 & 66.67 & 1 & 11.11 \\
\hline self-assessment & 0 & 00.0 & 7 & 70.00 & 3 & 30.00 \\
\hline $\begin{array}{c}\text { Indicator } \\
2.3 \text {. }\end{array}$ & \multicolumn{6}{|c|}{$\begin{array}{l}\text { Includes mother tongue (s) in } \\
\text { interactions with the child and } \\
\text { encourages families to use it in their } \\
\text { interactions with the child. }\end{array}$} \\
\hline Statements & \multicolumn{2}{|c|}{$\begin{array}{l}\text { Does not } \\
\text { apply }\end{array}$} & \multicolumn{2}{|c|}{$\begin{array}{l}\text { Sometim } \\
\text { es apply }\end{array}$} & \multicolumn{2}{|c|}{$\begin{array}{l}\text { Apply } \\
\text { always }\end{array}$} \\
\hline Results & \multicolumn{2}{|c|}{ Nr. $\%$} & \multicolumn{2}{|c|}{ Nr. $\quad \%$} & \multicolumn{2}{|c|}{ Nr. $\quad \%$} \\
\hline observations & 3 & 33.3 & 5 & 55.56 & 1 & 11.11 \\
\hline self-assessment & 4 & 40.0 & 3 & 30.00 & 3 & 30.00 \\
\hline $\begin{array}{c}\text { Indicator } \\
2.4 .\end{array}$ & \multicolumn{6}{|c|}{$\begin{array}{l}\text { It actively promotes the expression } \\
\text { of cultural identities. }\end{array}$} \\
\hline Statements & \multicolumn{2}{|c|}{$\begin{array}{l}\text { Does not } \\
\text { apply }\end{array}$} & \multicolumn{2}{|c|}{$\begin{array}{l}\text { Sometim } \\
\text { es apply }\end{array}$} & \multicolumn{2}{|c|}{$\begin{array}{l}\text { Apply } \\
\text { always }\end{array}$} \\
\hline Results & \multicolumn{2}{|c|}{ Nr. $\%$} & \multicolumn{2}{|c|}{ Nr. $\%$} & \multicolumn{2}{|c|}{ Nr. $\%$} \\
\hline observations & 3 & 33.3 & 2 & 22.22 & 4 & 44.44 \\
\hline self-assessment & 0 & 00.0 & 4 & 40.00 & 6 & 60.00 \\
\hline $\begin{array}{c}\text { Indicator } \\
2.5 \text {. }\end{array}$ & \multicolumn{6}{|c|}{$\begin{array}{l}\text { Supports the development of all the } \\
\text { identities of the child. }\end{array}$} \\
\hline Statements & \multicolumn{2}{|c|}{$\begin{array}{l}\text { Does not } \\
\text { apply }\end{array}$} & & $\begin{array}{l}\text { metim } \\
\text { apply }\end{array}$ & & $\begin{array}{l}\text { pply } \\
\text { ways }\end{array}$ \\
\hline Results & $\mathrm{Nr}$ & & & & $\mathrm{Nr}$. & \\
\hline observations & 2 & 22.2 & 1 & 11.11 & 6 & 66.67 \\
\hline self-assessment & 0 & 00.0 & 3 & 30.00 & 7 & 70.00 \\
\hline $\begin{array}{c}\text { Indicator } \\
2.6 \text {. }\end{array}$ & & $\begin{array}{l}\text { mon } \\
\text { ent } r \\
\text { ides. }\end{array}$ & & vithin $t$ & fa & $\begin{array}{l}\text { to the } \\
\text { iily that }\end{array}$ \\
\hline Statements & & $\begin{array}{l}\text { not } \\
\text { ply }\end{array}$ & & $\begin{array}{l}\text { metim } \\
\text { apply }\end{array}$ & & $\begin{array}{l}\text { pply } \\
\text { ways }\end{array}$ \\
\hline Results & $\mathrm{Nr}$ & $\%$ & $\mathrm{~N}$ & & $\mathrm{Nr}$. & \\
\hline observations & 3 & 33.3 & 0 & 00.00 & 6 & 66.67 \\
\hline self-a & 0 & 00.0 & 3 & 30.00 & 7 & 70.00 \\
\hline
\end{tabular}


Table 12. Principle 3: Promoting the sense of individuality, opinion and decisions of each child, motivating democratic values and practices.

\begin{tabular}{|c|c|c|c|c|c|c|}
\hline $\begin{array}{l}\text { Indicator } \\
3.1\end{array}$ & \multicolumn{6}{|c|}{$\begin{array}{l}\text { It offers great opportunities for } \\
\text { children to make choices. }\end{array}$} \\
\hline Statements & \multicolumn{2}{|c|}{$\begin{array}{l}\text { Does not } \\
\text { apply }\end{array}$} & \multicolumn{2}{|c|}{$\begin{array}{l}\text { Sometim } \\
\text { es apply }\end{array}$} & \multicolumn{2}{|c|}{$\begin{array}{l}\text { Apply } \\
\text { always }\end{array}$} \\
\hline Results & $\mathrm{N}$ & $\%$ & & & $\mathrm{Nr}$. & $\%$ \\
\hline observations & 2 & 22.2 & 2 & 22.22 & 6 & 66.67 \\
\hline self-assessment & 0 & 00.0 & & 00.00 & 10 & 100.00 \\
\hline $\begin{array}{l}\text { Indicator } \\
3.2 \text {. }\end{array}$ & \multicolumn{6}{|c|}{$\begin{array}{l}\text { Encourages children to express } \\
\text { themselves in numerous ways, } \\
\text { based on multiple language, gesture } \\
\text { and other non-verbal forms of } \\
\text { communication. }\end{array}$} \\
\hline Statements & \multicolumn{2}{|c|}{$\begin{array}{l}\text { Does not } \\
\text { apply }\end{array}$} & \multicolumn{2}{|c|}{$\begin{array}{l}\text { Sometim } \\
\text { es apply }\end{array}$} & \multicolumn{2}{|c|}{$\begin{array}{l}\text { Apply } \\
\text { always }\end{array}$} \\
\hline Results & \multicolumn{2}{|c|}{$\mathrm{Nr} . \quad \%$} & \multicolumn{2}{|c|}{ Nr. $\%$} & \multicolumn{2}{|c|}{ Nr. $\%$} \\
\hline observations & 2 & 22.2 & 6 & 66.67 & 1 & 11.11 \\
\hline self-assessment & 0 & 00.0 & 0 & 00.00 & 10 & 100.0 \\
\hline $\begin{array}{l}\text { Indicator } \\
3.3 \text {. }\end{array}$ & \multicolumn{6}{|c|}{$\begin{array}{l}\text { Supports parents in creative } \\
\text { listening and responding to children. }\end{array}$} \\
\hline Statements & \multicolumn{2}{|c|}{$\begin{array}{l}\text { Does not } \\
\text { apply }\end{array}$} & \multicolumn{2}{|c|}{$\begin{array}{l}\text { Sometim } \\
\text { es apply }\end{array}$} & \multicolumn{2}{|c|}{$\begin{array}{l}\text { Apply } \\
\text { always }\end{array}$} \\
\hline Results & \multicolumn{2}{|c|}{ Nr. $\%$} & \multicolumn{2}{|c|}{ Nr. $\%$} & \multicolumn{2}{|c|}{ Nr. $\%$} \\
\hline observations & 3 & 33.3 & 5 & 55.56 & 1 & 11.11 \\
\hline self-assessment & 0 & 00.0 & 0 & 00.00 & 10 & 100.00 \\
\hline $\begin{array}{l}\text { Indicator } \\
3.4 \text {. }\end{array}$ & \multicolumn{6}{|c|}{$\begin{array}{l}\text { Pricing, encouraging and looking } \\
\text { for the individual contribution of } \\
\text { children to experiences, knowledge } \\
\text { and expression in an open and } \\
\text { unbiased way. }\end{array}$} \\
\hline Statements & \multirow{2}{*}{\multicolumn{2}{|c|}{$\begin{array}{l}\text { Does not } \\
\text { apply } \\
\text { Nr. } \%\end{array}$}} & \multicolumn{2}{|c|}{$\begin{array}{l}\text { Sometim } \\
\text { es apply }\end{array}$} & \multicolumn{2}{|c|}{$\begin{array}{l}\text { Apply } \\
\text { always }\end{array}$} \\
\hline Results & & & \multicolumn{2}{|c|}{ Nr. $\%$} & \multicolumn{2}{|c|}{ Nr. $\%$} \\
\hline observations & 3 & 33.3 & 1 & 11.11 & 5 & 55.56 \\
\hline self-assessment & 0 & 00.0 & 1 & 10.00 & 9 & 90.00 \\
\hline $\begin{array}{l}\text { Indicator } \\
3.5 \text {. }\end{array}$ & \multicolumn{6}{|c|}{$\begin{array}{l}\text { It implements effective positive } \\
\text { guiding strategies that support the } \\
\text { restoration of justice and the } \\
\text { preservation of dignity. }\end{array}$} \\
\hline Statements & \multicolumn{2}{|c|}{$\begin{array}{c}\text { Does not } \\
\text { apply }\end{array}$} & \multicolumn{2}{|c|}{$\begin{array}{l}\text { Sometim } \\
\text { es apply }\end{array}$} & \multicolumn{2}{|c|}{$\begin{array}{l}\text { Apply } \\
\text { always }\end{array}$} \\
\hline Results & \multicolumn{2}{|c|}{ Nr. $\%$} & \multicolumn{2}{|c|}{ Nr. $\%$} & \multicolumn{2}{|c|}{ Nr. $\%$} \\
\hline observations & 4 & 44.4 & 5 & 55.56 & 0 & 00.00 \\
\hline self-assessment & 0 & 00.0 & 0 & 00.00 & 10 & 100.00 \\
\hline
\end{tabular}

Table 13. Principle 4: Inclusion is promoted through partnerships with families.

\begin{tabular}{|c|c|c|c|c|c|c|}
\hline $\begin{array}{c}\text { Indicator } \\
4.1\end{array}$ & \multicolumn{6}{|c|}{$\begin{array}{l}\text { Dialogue with families about how } \\
\text { services can be improved to show } \\
\text { respect and appreciate diversity. }\end{array}$} \\
\hline Statements & \multirow{2}{*}{\multicolumn{2}{|c|}{$\begin{array}{l}\text { Does not } \\
\text { apply } \\
\text { Nr. } \%\end{array}$}} & \multicolumn{2}{|c|}{$\begin{array}{l}\text { Sometim } \\
\text { es apply }\end{array}$} & \multicolumn{2}{|c|}{$\begin{array}{l}\text { Apply } \\
\text { always }\end{array}$} \\
\hline Results & & & & & & \\
\hline observations & 4 & 44.4 & 5 & 55.56 & 0 & 00.00 \\
\hline self-assessment & 0 & 00.0 & 2 & 20.00 & 8 & 80.00 \\
\hline $\begin{array}{c}\text { Indicator } \\
4.2 \text {. }\end{array}$ & \multicolumn{6}{|c|}{$\begin{array}{l}\text { Dialogue with families about how } \\
\text { services can be more inclusive, } \\
\text { including advocacy and support for } \\
\text { families with children with special } \\
\text { needs. }\end{array}$} \\
\hline Statements & \multicolumn{2}{|c|}{$\begin{array}{c}\text { Does not } \\
\text { apply }\end{array}$} & \multicolumn{2}{|c|}{$\begin{array}{l}\text { Sometim } \\
\text { es apply }\end{array}$} & \multicolumn{2}{|c|}{$\begin{array}{l}\text { Apply } \\
\text { always }\end{array}$} \\
\hline Results & \multicolumn{2}{|c|}{ Nr. $\%$} & \multicolumn{2}{|c|}{ Nr. $\%$} & \multicolumn{2}{|c|}{ Nr. $\quad \%$} \\
\hline observations & 3 & 33.3 & 1 & 11.11 & 5 & 55.56 \\
\hline self-assessment & 0 & 00.0 & 3 & 30.00 & 7 & 70.00 \\
\hline $\begin{array}{c}\text { Indicator } \\
4.3 .\end{array}$ & \multicolumn{6}{|c|}{$\begin{array}{l}\text { Work with families and specialists } \\
\text { to create an inclusion plan. } \\
\text { Discusses in this plan the successes } \\
\text { and challenges, observations and } \\
\text { reflections related to the individual } \\
\text { work with the child. }\end{array}$} \\
\hline Statements & \multicolumn{2}{|c|}{$\begin{array}{c}\text { Does not } \\
\text { apply }\end{array}$} & \multicolumn{2}{|c|}{$\begin{array}{l}\text { Sometim } \\
\text { es apply }\end{array}$} & \multicolumn{2}{|c|}{$\begin{array}{l}\text { Apply } \\
\text { always }\end{array}$} \\
\hline Results & $\mathrm{N}_{1}$ & $\%$ & $\mathrm{~N}$ & & N & \\
\hline observations & 3 & 33.3 & 6 & 66.67 & 0 & 00.00 \\
\hline self-assessment & 4 & 40.0 & 3 & 30.00 & 3 & 30.00 \\
\hline
\end{tabular}




\subsection{Priority area "Health, well-being, nutrition"}

Table 14. Principle 1: Every child's health is encouraged.

\begin{tabular}{|c|c|c|c|c|c|c|}
\hline $\begin{array}{c}\text { Indicator } \\
1.1 .\end{array}$ & \multicolumn{6}{|c|}{$\begin{array}{l}\text { Providing a safe environment that } \\
\text { promotes development and } \\
\text { well-being. }\end{array}$} \\
\hline Statements & \multicolumn{2}{|c|}{$\begin{array}{c}\text { Does not } \\
\text { apply }\end{array}$} & \multicolumn{2}{|c|}{$\begin{array}{l}\text { Sometim } \\
\text { es apply }\end{array}$} & \multicolumn{2}{|c|}{$\begin{array}{l}\text { Apply } \\
\text { always }\end{array}$} \\
\hline Results & \multicolumn{2}{|c|}{ Nr. $\%$} & \multicolumn{2}{|c|}{ Nr. $\%$} & \multicolumn{2}{|c|}{ Nr. $\%$} \\
\hline observations & 0 & 00.0 & 3 & 33.33 & 6 & 66.67 \\
\hline self-assessment & 0 & 00.0 & 0 & 00.00 & 10 & 100.0 \\
\hline $\begin{array}{c}\text { Indicator } \\
1.2\end{array}$ & \multicolumn{6}{|c|}{$\begin{array}{l}\text { Promoting and maintaining effective } \\
\text { hygiene practices that control the } \\
\text { spread of infectious diseases and } \\
\text { support healthy habits. }\end{array}$} \\
\hline Statements & \multicolumn{2}{|c|}{$\begin{array}{c}\text { Does not } \\
\text { apply }\end{array}$} & \multicolumn{2}{|c|}{$\begin{array}{l}\text { Sometim } \\
\text { es apply }\end{array}$} & \multicolumn{2}{|c|}{$\begin{array}{l}\text { Apply } \\
\text { always }\end{array}$} \\
\hline Results & \multicolumn{2}{|c|}{ Nr. $\%$} & \multicolumn{2}{|c|}{ Nr. $\%$} & \multicolumn{2}{|c|}{ Nr. $\quad \%$} \\
\hline observations & 1 & 11.1 & 2 & 22.22 & 7 & 77.78 \\
\hline self-assessment & 0 & 00.0 & 0 & 00.00 & 10 & 100.0 \\
\hline $\begin{array}{c}\text { Indicator } \\
1.3 .\end{array}$ & \multicolumn{6}{|c|}{$\begin{array}{l}\text { Immunization information and } \\
\text { timetables are provided to } \\
\text { encourage parents to immunize their } \\
\text { children in a timely manner. }\end{array}$} \\
\hline Statements & \multirow{2}{*}{\multicolumn{2}{|c|}{$\begin{array}{c}\text { Does not } \\
\text { apply }\end{array}$}} & \multirow{2}{*}{\multicolumn{2}{|c|}{$\begin{array}{r}\text { Sometim } \\
\text { es apply } \\
\text { Nr. } \%\end{array}$}} & \multicolumn{2}{|c|}{$\begin{array}{c}\text { Apply } \\
\text { always }\end{array}$} \\
\hline Results & & & & & $\mathrm{Nr}$. & \\
\hline observations & 2 & 22.2 & 6 & 66.67 & 1 & 11.11 \\
\hline self-as & 0 & 00.0 & 0 & 00.00 & 10 & 100.0 \\
\hline $\begin{array}{c}\text { Indicator } \\
1.4 .\end{array}$ & \multicolumn{6}{|c|}{$\begin{array}{l}\text { There are procedures to prevent and } \\
\text { deal with injuries that include first } \\
\text { aid, child's heartbeat, and home and } \\
\text { offsite safety education. }\end{array}$} \\
\hline Statements & \multicolumn{2}{|c|}{$\begin{array}{c}\text { Does not } \\
\text { apply }\end{array}$} & \multicolumn{2}{|c|}{$\begin{array}{l}\text { Sometim } \\
\text { es apply }\end{array}$} & \multicolumn{2}{|c|}{$\begin{array}{l}\text { Apply } \\
\text { always }\end{array}$} \\
\hline Results & \multicolumn{2}{|c|}{$\mathrm{Nr} . \%$} & \multicolumn{2}{|c|}{ Nr. $\%$} & \multicolumn{2}{|c|}{ Nr. $\quad \%$} \\
\hline observations & 2 & 22.2 & 6 & 66.67 & 1 & 11.11 \\
\hline self-assessment & 0 & 00.0 & 0 & 00.00 & 10 & 100.0 \\
\hline $\begin{array}{c}\text { Indicator } \\
1.5 .\end{array}$ & \multicolumn{6}{|c|}{$\begin{array}{l}\text { The need for regular health checks } \\
\text { and monitoring of their outcomes is } \\
\text { underlined. }\end{array}$} \\
\hline Statements & \multicolumn{2}{|c|}{$\begin{array}{c}\text { Does not } \\
\text { apply }\end{array}$} & \multirow{2}{*}{\multicolumn{2}{|c|}{$\begin{array}{l}\text { Sometim } \\
\text { es apply }\end{array}$}} & \multicolumn{2}{|c|}{$\begin{array}{l}\text { Apply } \\
\text { always }\end{array}$} \\
\hline Results & \multicolumn{2}{|c|}{$\mathrm{Nr} . \quad \%$} & & & $\mathrm{Jr}$. & \\
\hline observations & 2 & 22.2 & 6 & 66.6 & 1 & 11.11 \\
\hline self-assessment & 0 & 00.0 & 1 & 10.0 & 9 & 90.00 \\
\hline $\begin{array}{c}\text { Indicator } \\
1.6 .\end{array}$ & & $\begin{array}{l}\text { atisfies } \\
\text { ical ac }\end{array}$ & & $\begin{array}{l}\text { chil } \\
\text {, as } \mathrm{x}\end{array}$ & & $\begin{array}{l}\text { ed for } \\
\text { rest and }\end{array}$ \\
\hline Statements & & $\begin{array}{l}\text { not } \\
\text { bly }\end{array}$ & & $\begin{array}{l}\text { netim } \\
\text { apply }\end{array}$ & & $\begin{array}{l}\text { pply } \\
\text { ways }\end{array}$ \\
\hline Results & $\mathrm{Nr}$ & $\%$ & & & $\mathrm{Nr}$. & \\
\hline observations & 1 & 11.1 & 2 & 22.22 & 6 & 66.67 \\
\hline self-asse & 0 & 00.0 & 0 & 00.00 & 10 & 100.0 \\
\hline
\end{tabular}

Table 15. Principle 2: Meeting the nutritional needs of the child.

\begin{tabular}{|c|c|c|c|c|c|c|}
\hline $\begin{array}{c}\text { Indicator } \\
2.1 .\end{array}$ & \multicolumn{6}{|c|}{$\begin{array}{l}\text { Breastfeeding is supported and } \\
\text { information about it and the } \\
\text { conditions facilitating it are } \\
\text { provided. }\end{array}$} \\
\hline Statements & \multicolumn{2}{|c|}{$\begin{array}{c}\text { Does not } \\
\text { apply }\end{array}$} & \multicolumn{2}{|c|}{$\begin{array}{l}\text { Sometim } \\
\text { es apply }\end{array}$} & \multicolumn{2}{|c|}{$\begin{array}{l}\text { Apply } \\
\text { always }\end{array}$} \\
\hline Results & \multicolumn{2}{|c|}{ Nr. $\%$} & \multicolumn{2}{|c|}{ Nr. $\%$} & \multicolumn{2}{|c|}{ Nr. $\%$} \\
\hline observations & 4 & 44.4 & 5 & 55.56 & 0 & 00.00 \\
\hline self-asses & 0 & 00.0 & 2 & 20.00 & 8 & 80.00 \\
\hline $\begin{array}{c}\text { Indicator } \\
2.2 \text {. }\end{array}$ & \multicolumn{6}{|c|}{$\begin{array}{l}\text { Support (including through } \\
\text { modeling) healthy eating habits for } \\
\text { young children; there is a choice of } \\
\text { healthy, age-appropriate foods that } \\
\text { are culturally and regionally } \\
\text { tailored. }\end{array}$} \\
\hline Statements & \multicolumn{2}{|c|}{$\begin{array}{c}\text { Does not } \\
\text { apply }\end{array}$} & \multicolumn{2}{|c|}{$\begin{array}{l}\text { Sometim } \\
\text { es apply }\end{array}$} & \multicolumn{2}{|c|}{$\begin{array}{l}\text { Apply } \\
\text { always }\end{array}$} \\
\hline Results & \multicolumn{2}{|c|}{ Nr. $\%$} & \multicolumn{2}{|c|}{ Nr. $\quad \%$} & \multicolumn{2}{|c|}{ Nr. $\quad \%$} \\
\hline observations & 1 & 11.1 & 6 & 66.67 & 2 & 22.22 \\
\hline self-assessment & 0 & 00.0 & 0 & 00.00 & 10 & 100.00 \\
\hline $\begin{array}{c}\text { Indicator } \\
2.3\end{array}$ & \multicolumn{6}{|c|}{$\begin{array}{l}\text { Ensure that food meets the dietary } \\
\text { requirements of children and takes } \\
\text { into account allergies to certain } \\
\text { foods. }\end{array}$} \\
\hline Statements & \multicolumn{2}{|c|}{$\begin{array}{c}\text { Does not } \\
\text { apply }\end{array}$} & \multirow{2}{*}{\multicolumn{2}{|c|}{$\begin{array}{l}\text { Sometim } \\
\text { es apply } \\
\mathrm{Nr} \%\end{array}$}} & \multicolumn{2}{|c|}{$\begin{array}{l}\text { Apply } \\
\text { always }\end{array}$} \\
\hline Results & \multicolumn{2}{|c|}{ Nr. $\%$} & & & \multicolumn{2}{|c|}{ Nr. $\%$} \\
\hline observations & 0 & 00.0 & 7 & 77.78 & 2 & 22.22 \\
\hline self-assessment & 0 & 00.0 & 0 & 00.00 & 10 & 100.00 \\
\hline $\begin{array}{c}\text { Indicator } \\
2.4\end{array}$ & \multicolumn{6}{|c|}{$\begin{array}{l}\text { Hygienic procedures for storing, } \\
\text { preparing and serving food are } \\
\text { followed and encouraged. }\end{array}$} \\
\hline Statements & \multicolumn{2}{|c|}{$\begin{array}{l}\text { Does not } \\
\text { apply } \\
\text { Nr. } \%\end{array}$} & \multicolumn{2}{|c|}{$\begin{array}{r}\text { Sometim } \\
\text { es apply } \\
\text { Nr. } \%\end{array}$} & \multicolumn{2}{|c|}{$\begin{array}{l}\text { Apply } \\
\text { always }\end{array}$} \\
\hline observations & . & & 7 & & & \\
\hline self-assessment & 0 & 00.0 & 7 & 77.78 & 2 & 22.22 \\
\hline $\begin{array}{c}\text { Indicator } \\
2.5 \text {. }\end{array}$ & & $\begin{array}{l}\text { archin } \\
\text { lies ( } \\
\text { hygi } \\
\text { ided }\end{array}$ & & $\begin{array}{l}\text { nd di } \\
\text { their s } \\
\text { practi } \\
\text { arly c }\end{array}$ & & $\begin{array}{l}\text { g with } \\
\text { ), food } \\
\text { lat are } \\
\text { d care }\end{array}$ \\
\hline Statements & & $\begin{array}{l}\text { s not } \\
\text { ply }\end{array}$ & & $\begin{array}{l}\text { metim } \\
\text { apply }\end{array}$ & & $\begin{array}{l}\text { pply } \\
\text { ways }\end{array}$ \\
\hline Results & $\mathrm{N}_{1}$ & $\%$ & $\mathrm{~N}_{1}$ & & $\mathrm{~N}_{1}$ & \\
\hline rvations & 3 & 33.3 & 0 & 00.00 & 6 & 66.67 \\
\hline self-assessment & 0 & 00.0 & 5 & 50.00 & 5 & 50.00 \\
\hline
\end{tabular}


Table 16. Principle 3: Health care and nutrition are a source of pleasure and effection.

\begin{tabular}{|c|c|c|c|c|c|c|}
\hline $\begin{array}{c}\text { Indicator } \\
3.1 .\end{array}$ & \multicolumn{6}{|c|}{$\begin{array}{l}\text { Promoting nutrition and care as a } \\
\text { source of pleasure as part of the } \\
\text { relationship between care and } \\
\text { learning. }\end{array}$} \\
\hline Statements & \multicolumn{2}{|c|}{$\begin{array}{c}\text { Does not } \\
\text { apply }\end{array}$} & \multicolumn{2}{|c|}{$\begin{array}{l}\text { Sometim } \\
\text { es apply }\end{array}$} & \multicolumn{2}{|c|}{$\begin{array}{l}\text { Apply } \\
\text { always }\end{array}$} \\
\hline Results & \multicolumn{2}{|c|}{$\mathrm{Nr} . \quad \%$} & \multicolumn{2}{|c|}{ Nr. $\%$} & \multicolumn{2}{|c|}{ Nr. $\quad \%$} \\
\hline observations & 2 & 22.2 & 7 & 77.78 & 0 & 00.00 \\
\hline self-asse & 0 & 00.0 & 0 & 00.00 & 10 & 100.00 \\
\hline $\begin{array}{l}\text { Indicator } \\
3.2 \text {. }\end{array}$ & \multicolumn{6}{|c|}{$\begin{array}{l}\text { The active participation of the child } \\
\text { in these activities is encouraged. }\end{array}$} \\
\hline Statements & \multicolumn{2}{|c|}{$\begin{array}{l}\text { Does not } \\
\text { apply }\end{array}$} & \multicolumn{2}{|c|}{$\begin{array}{l}\text { Sometim } \\
\text { es apply }\end{array}$} & \multicolumn{2}{|c|}{$\begin{array}{l}\text { Apply } \\
\text { always }\end{array}$} \\
\hline Results & \multicolumn{2}{|c|}{$\mathrm{Nr} . \quad \%$} & \multicolumn{2}{|c|}{ Nr. $\%$} & \multicolumn{2}{|c|}{ Nr. $\%$} \\
\hline observations & 1 & 11.1 & 3 & 33.33 & 6 & 66.67 \\
\hline self-assessment & 1 & 11.1 & 3 & 33.33 & 6 & 66.67 \\
\hline $\begin{array}{c}\text { Indicator } \\
3.3 \text {. }\end{array}$ & \multicolumn{6}{|c|}{$\begin{array}{l}\text { Activities related to nutrition and } \\
\text { care that meet the individual needs } \\
\text { of children are provided and } \\
\text { encouraged. }\end{array}$} \\
\hline Statements & \multirow{2}{*}{\multicolumn{2}{|c|}{$\begin{array}{l}\text { Does not } \\
\text { apply } \\
\text { Nr. } \%\end{array}$}} & \multicolumn{2}{|c|}{$\begin{array}{l}\text { Sometim } \\
\text { es apply }\end{array}$} & \multicolumn{2}{|c|}{$\begin{array}{c}\text { Apply } \\
\text { always }\end{array}$} \\
\hline Results & & & \multicolumn{2}{|c|}{ Nr. $\%$} & \multicolumn{2}{|c|}{ Nr. $\%$} \\
\hline observatio & 3 & 33.3 & 5 & 55.56 & 1 & 11.11 \\
\hline self-assessn & 0 & 00.0 & 0 & 00.00 & 10 & 100.00 \\
\hline $\begin{array}{c}\text { Indicator } \\
3.4 \text {. }\end{array}$ & \multicolumn{6}{|c|}{$\begin{array}{l}\text { There are close and positive } \\
\text { interactions during nutrition and } \\
\text { care that enhance attachment. }\end{array}$} \\
\hline Statements & \multicolumn{2}{|c|}{$\begin{array}{c}\text { Does not } \\
\text { apply }\end{array}$} & \multicolumn{2}{|c|}{$\begin{array}{l}\text { Sometim } \\
\text { es apply }\end{array}$} & \multicolumn{2}{|c|}{$\begin{array}{l}\text { Apply } \\
\text { always }\end{array}$} \\
\hline Results & \multicolumn{2}{|c|}{ Nr. $\%$} & \multicolumn{2}{|c|}{ Nr. $\%$} & \multicolumn{2}{|c|}{ Nr. $\%$} \\
\hline observations & 2 & 22.2 & 6 & 66.67 & 1 & 11.11 \\
\hline self-assessment & 0 & 00.0 & 4 & 40.00 & 6 & 60.00 \\
\hline
\end{tabular}

Table 17. Principle 4: Every child is protected from violence, lack of care and injury by promoting appropriate practices, prevention and intervention.

\begin{tabular}{|c|c|c|c|c|c|c|}
\hline $\begin{array}{c}\text { Indicator } \\
4.1 .\end{array}$ & \multicolumn{6}{|c|}{$\begin{array}{l}\text { The specialist monitors the mental } \\
\text { and physical condition of children, } \\
\text { identifies signs of violence and / or } \\
\text { lack of care that affect children's } \\
\text { health and informs relevant } \\
\text { authorities. }\end{array}$} \\
\hline Statements & \multirow{2}{*}{\multicolumn{2}{|c|}{$\begin{array}{l}\text { Does not } \\
\text { apply } \\
\mathrm{Nr} . \%\end{array}$}} & \multicolumn{2}{|c|}{$\begin{array}{l}\text { Sometim } \\
\text { es apply }\end{array}$} & \multicolumn{2}{|c|}{$\begin{array}{l}\text { Apply } \\
\text { always }\end{array}$} \\
\hline Results & & & \multicolumn{2}{|c|}{ Nr. $\%$} & \multicolumn{2}{|c|}{ Nr. $\%$} \\
\hline observations & 1 & 11.1 & 2 & 22.22 & 6 & 66.67 \\
\hline gelf-assessment & 0 & 00.0 & 8 & 80.00 & 2 & 20.00 \\
\hline $\begin{array}{c}\text { Indicator } \\
4.2 .\end{array}$ & \multicolumn{6}{|c|}{$\begin{array}{l}\text { The specialist follows established } \\
\text { procedures for recommendations } \\
\text { and work with families when } \\
\text { children show signs of atypical } \\
\text { behavior, depression or anxiety. }\end{array}$} \\
\hline Statements & \multirow{2}{*}{\multicolumn{2}{|c|}{$\begin{array}{l}\text { Does not } \\
\text { apply } \\
\mathrm{Nr} . \%\end{array}$}} & \multirow{2}{*}{\multicolumn{2}{|c|}{$\begin{array}{l}\text { Sometim } \\
\text { es apply } \\
\text { Nr. } \%\end{array}$}} & \multirow{2}{*}{\multicolumn{2}{|c|}{$\begin{array}{c}\text { Apply } \\
\text { always }\end{array}$}} \\
\hline Results & & & & & & \\
\hline observations & 3 & 33.3 & 6 & 66.67 & 0 & 00.00 \\
\hline gelf-assesgment & 0 & 00.0 & 4 & 40.00 & 6 & 60.00 \\
\hline $\begin{array}{c}\text { Indicator } \\
4.3 .\end{array}$ & \multicolumn{6}{|c|}{$\begin{array}{l}\text { The specialist maintains families } \\
\text { during a crisis, stress, and maternal } \\
\text { or depression. }\end{array}$} \\
\hline Statements & \multirow{2}{*}{\multicolumn{2}{|c|}{$\begin{array}{l}\text { Does not } \\
\text { apply } \\
\mathrm{Nr} . \%\end{array}$}} & \multirow{2}{*}{\multicolumn{2}{|c|}{$\begin{array}{l}\text { Sometim } \\
\text { es apply } \\
\mathrm{Nr} . \%\end{array}$}} & \multirow{2}{*}{\multicolumn{2}{|c|}{$\begin{array}{c}\text { Apply } \\
\text { always } \\
\text { Nr. } \% \\
\end{array}$}} \\
\hline Results & & & & & & \\
\hline observations & 9 & 100 & 0 & 00.00 & 0 & 00.00 \\
\hline self-assesgment & 2 & 20.0 & 8 & 80.00 & 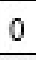 & 00.00 \\
\hline $\begin{array}{c}\text { Indicator } \\
4.4 .\end{array}$ & \multicolumn{6}{|c|}{$\begin{array}{l}\text { The specialist knows the roles, legal } \\
\text { responsibilities and procedures to } \\
\text { protect children at risk of violence } \\
\text { and / or lack of care, including } \\
\text { knowledge of how to keep in touch } \\
\text { with a family suspected of violence } \\
\text { against the child. }\end{array}$} \\
\hline Statements & \multirow{2}{*}{\multicolumn{2}{|c|}{$\begin{array}{c}\text { Does not } \\
\text { apply }\end{array}$}} & \multicolumn{2}{|c|}{$\begin{array}{l}\text { Sometim } \\
\text { es apply }\end{array}$} & \multicolumn{2}{|c|}{$\begin{array}{l}\text { Apply } \\
\text { always }\end{array}$} \\
\hline Results & & & & & & \\
\hline observations & 4 & 44.4 & 3 & 33.33 & 2 & 22.22 \\
\hline & 1 & 10.0 & 7 & 70.00 & 2 & 20.00 \\
\hline
\end{tabular}




\subsection{Priority area "Development and Learning"}

Table 18. Principle 1: The development of children is holistically approached.

\begin{tabular}{|c|c|c|c|c|c|c|}
\hline $\begin{array}{c}\text { Indicator } \\
1.1 .\end{array}$ & \multicolumn{6}{|c|}{$\begin{array}{l}\text { The specialist should follow and / or } \\
\text { develop a curriculum that covers all } \\
\text { areas of experience in the child's } \\
\text { holistic development. }\end{array}$} \\
\hline Statements & \multirow{2}{*}{\multicolumn{2}{|c|}{$\begin{array}{l}\text { Does not } \\
\text { apply } \\
\text { Nr. } \%\end{array}$}} & \multirow{2}{*}{\multicolumn{2}{|c|}{$\begin{array}{l}\text { Sometim } \\
\text { es apply } \\
\text { Nr. } \%\end{array}$}} & \multicolumn{2}{|c|}{$\begin{array}{l}\text { Apply } \\
\text { always }\end{array}$} \\
\hline Results & & & & & & \\
\hline observations & 0 & 00.0 & 4 & 44.44 & 5 & 55.56 \\
\hline self-assessment & 0 & 00.0 & 1 & 10.00 & 9 & 90.00 \\
\hline $\begin{array}{c}\text { Indicator } \\
1.2 .\end{array}$ & \multicolumn{6}{|c|}{$\begin{array}{l}\text { The specialist integrates the child's } \\
\text { previous experience in introducing } \\
\text { new experiences. }\end{array}$} \\
\hline Statements & \multicolumn{2}{|c|}{$\begin{array}{c}\text { Does not } \\
\text { apply }\end{array}$} & \multirow{2}{*}{\multicolumn{2}{|c|}{$\begin{array}{r}\text { Sometim } \\
\text { es apply } \\
\text { Nr. } \%\end{array}$}} & \multicolumn{2}{|c|}{$\begin{array}{l}\text { Apply } \\
\text { always }\end{array}$} \\
\hline Results & \multicolumn{2}{|c|}{ Nr. $\%$} & & & \multicolumn{2}{|c|}{ Nr. $\%$} \\
\hline observations & 0 & 00.0 & 2 & 22.22 & 7 & 77.78 \\
\hline self-asse & 0 & 00.0 & 1 & 10.00 & 9 & 90.00 \\
\hline $\begin{array}{c}\text { Indicator } \\
1.3 .\end{array}$ & \multicolumn{6}{|c|}{$\begin{array}{l}\text { The specialist encourages and uses } \\
\text { diverse approaches to learning } \\
\text { children. }\end{array}$} \\
\hline Statements & \multirow{2}{*}{\multicolumn{2}{|c|}{$\begin{array}{l}\text { Does not } \\
\text { apply } \\
\text { Nr. } \%\end{array}$}} & \multicolumn{2}{|c|}{$\begin{array}{l}\text { Sometim } \\
\text { es apply }\end{array}$} & \multicolumn{2}{|c|}{$\begin{array}{l}\text { Apply } \\
\text { always }\end{array}$} \\
\hline Results & & & \multicolumn{2}{|c|}{ Nr. $\%$} & \multicolumn{2}{|c|}{ Nr. $\quad \%$} \\
\hline observations & 3 & 33.3 & 0 & 00.00 & 6 & 66.67 \\
\hline self-assessment & 0 & 00.0 & 0 & 00.00 & 10 & 100.00 \\
\hline $\begin{array}{c}\text { Indicator } \\
1.4 .\end{array}$ & \multicolumn{6}{|c|}{$\begin{array}{l}\text { The specialist offers and encourages } \\
\text { a combination of varied and } \\
\text { predictable experiences that } \\
\text { encourage study, experimentation, } \\
\text { independent research, and the child's } \\
\text { creativity. }\end{array}$} \\
\hline Statements & \multirow{2}{*}{\multicolumn{2}{|c|}{$\begin{array}{l}\text { Does not } \\
\text { apply } \\
\text { Nr. } \%\end{array}$}} & \multicolumn{2}{|c|}{$\begin{array}{l}\text { Sometim } \\
\text { es apply }\end{array}$} & \multicolumn{2}{|c|}{$\begin{array}{l}\text { Apply } \\
\text { always }\end{array}$} \\
\hline Results & & & & & $\mathrm{N}_{1}$ & $\%$ \\
\hline observations & 2 & 22.2 & 1 & 11.11 & 6 & 66.67 \\
\hline self-assessment & 0 & 00.0 & 7 & 70.00 & 3 & 30.00 \\
\hline
\end{tabular}

Table 19. Principle 2: Care is seen as an opportunity for development and learning.

\begin{tabular}{|c|c|c|c|c|c|c|}
\hline $\begin{array}{c}\text { Indicator } \\
2.1\end{array}$ & \multicolumn{6}{|c|}{$\begin{array}{l}\text { Daily nutrition and health care are } \\
\text { encouraged to stimulate children's } \\
\text { active participation and autonomy. }\end{array}$} \\
\hline Statements & \multirow{2}{*}{\multicolumn{2}{|c|}{$\begin{array}{l}\text { Does not } \\
\text { apply } \\
\text { Nr. } \%\end{array}$}} & \multirow{2}{*}{\multicolumn{2}{|c|}{$\begin{array}{l}\text { Sometim } \\
\text { es apply } \\
\text { Nr. } \%\end{array}$}} & \multicolumn{2}{|c|}{$\begin{array}{l}\text { Apply } \\
\text { always }\end{array}$} \\
\hline Results & & & & & Nr. & \\
\hline observations & 0 & 00.0 & 2 & 22.22 & 7 & 77.78 \\
\hline self-assessment & 0 & 00.0 & 0 & 00.00 & 10 & 100.00 \\
\hline $\begin{array}{c}\text { Indicator } \\
2.2 \text {. }\end{array}$ & \multicolumn{6}{|c|}{$\begin{array}{l}\text { Care and transitions are encouraged } \\
\text { that encourage the child to discuss } \\
\text { and collaborate in advance, taking } \\
\text { into account the child's mood or } \\
\text { attention. }\end{array}$} \\
\hline Statements & \multicolumn{2}{|c|}{$\begin{array}{l}\text { Does not } \\
\text { apply }\end{array}$} & \multicolumn{2}{|c|}{$\begin{array}{l}\text { Sometim } \\
\text { es apply }\end{array}$} & \multicolumn{2}{|c|}{$\begin{array}{l}\text { Apply } \\
\text { always }\end{array}$} \\
\hline Results & \multicolumn{2}{|c|}{ Nr. $\%$} & \multicolumn{2}{|c|}{ Nr. $\quad \%$} & \multicolumn{2}{|c|}{ Nr. $\%$} \\
\hline observations & 2 & 22.2 & 7 & 77.78 & 0 & 00.00 \\
\hline self-assessment & 6 & 60.0 & 2 & 20.00 & 2 & 20.00 \\
\hline $\begin{array}{c}\text { Indicator } \\
2.3\end{array}$ & \multicolumn{6}{|c|}{$\begin{array}{l}\text { Opportunities are being used to } \\
\text { encourage child development in an } \\
\text { integrated way in the context of } \\
\text { naturally occurring events. }\end{array}$} \\
\hline Statements & \multirow{2}{*}{\multicolumn{2}{|c|}{$\begin{array}{l}\text { Does not } \\
\text { apply } \\
\text { Nr. } \%\end{array}$}} & \multicolumn{2}{|c|}{$\begin{array}{l}\text { Sometim } \\
\text { es apply }\end{array}$} & \multicolumn{2}{|c|}{$\begin{array}{l}\text { Apply } \\
\text { always }\end{array}$} \\
\hline Results & & & $\mathrm{Nr}$ & $\%$ & Nr. & $\%$ \\
\hline observations & 2 & 22.2 & 7 & 77.78 & 0 & 00.00 \\
\hline self-assessment & 0 & 00.0 & 5 & 50.00 & 5 & 50.00 \\
\hline $\begin{array}{c}\text { Indicator } \\
2.4 .\end{array}$ & \multicolumn{6}{|c|}{$\begin{array}{l}\text { Caring activities are used to talk to } \\
\text { the child. }\end{array}$} \\
\hline Statements & \multicolumn{2}{|c|}{$\begin{array}{l}\text { Does not } \\
\text { apply }\end{array}$} & \multicolumn{2}{|c|}{$\begin{array}{l}\text { Sometim } \\
\text { es apply }\end{array}$} & \multicolumn{2}{|c|}{$\begin{array}{l}\text { Apply } \\
\text { always }\end{array}$} \\
\hline Results & $\mathrm{N}$ & $\%$ & $\mathrm{Nr}$ & & Nr. & \\
\hline observations & 1 & 11.1 & 3 & 33.33 & 5 & 55.55 \\
\hline self-assessment & 0 & 00.0 & 0 & 00.00 & 10 & 100.00 \\
\hline
\end{tabular}


Table 20. Principle 3: Play is a source and strategy for development, well-being and learning.

\begin{tabular}{|c|c|c|c|c|c|c|}
\hline $\begin{array}{c}\text { Indicator } \\
3.1 .\end{array}$ & \multicolumn{6}{|c|}{$\begin{array}{l}\text { The specialist encourages the game } \\
\text { as a source of development, } \\
\text { exploration, discovery, engagement } \\
\text { and joy. }\end{array}$} \\
\hline Statements & \multirow{2}{*}{\multicolumn{2}{|c|}{$\begin{array}{c}\text { Does not } \\
\text { apply } \\
\text { Nr. } \quad \%\end{array}$}} & \multirow{2}{*}{\multicolumn{2}{|c|}{$\begin{array}{r}\text { Sometim } \\
\text { es apply } \\
\text { Nr. } \%\end{array}$}} & \multicolumn{2}{|c|}{$\begin{array}{l}\text { Apply } \\
\text { always }\end{array}$} \\
\hline Results & & & & & $\mathrm{Nr}$. & \\
\hline observations & 1 & 11.1 & 1 & 11.11 & 7 & 77.78 \\
\hline self-assessment & 0 & 00.0 & 0 & 00.00 & 10 & 100.00 \\
\hline $\begin{array}{c}\text { Indicator } \\
3.2 \text {. }\end{array}$ & \multicolumn{6}{|c|}{$\begin{array}{l}\text { The specialist encourages the } \\
\text { development and age-appropriate, } \\
\text { gaming experience based on the } \\
\text { knowledge of each child. }\end{array}$} \\
\hline Statements & \multicolumn{2}{|c|}{$\begin{array}{c}\text { Does not } \\
\text { apply }\end{array}$} & \multicolumn{2}{|c|}{$\begin{array}{l}\text { Sometim } \\
\text { es apply }\end{array}$} & \multicolumn{2}{|c|}{$\begin{array}{l}\text { Apply } \\
\text { always }\end{array}$} \\
\hline Results & \multicolumn{2}{|c|}{ Nr. $\%$} & \multicolumn{2}{|c|}{ Nr. $\%$} & \multicolumn{2}{|c|}{ Nr. $\%$} \\
\hline observations & 2 & 22.2 & 1 & 11.11 & 6 & 66.67 \\
\hline self-asses & 0 & 00.0 & 0 & 00.00 & 10 & 100.00 \\
\hline $\begin{array}{c}\text { Indicator } \\
3.3 .\end{array}$ & \multicolumn{6}{|c|}{$\begin{array}{l}\text { The specialist realizes the role of the } \\
\text { key adults they play in the game and } \\
\text { the importance of being close to the } \\
\text { child during these experiences. }\end{array}$} \\
\hline Statements & \multirow{2}{*}{\multicolumn{2}{|c|}{$\begin{array}{l}\text { Does not } \\
\text { apply } \\
\text { Nr. } \%\end{array}$}} & \multirow{2}{*}{\multicolumn{2}{|c|}{$\begin{array}{l}\text { Sometim } \\
\text { es apply } \\
\text { Nr. } \%\end{array}$}} & \multicolumn{2}{|c|}{$\begin{array}{l}\text { Apply } \\
\text { always }\end{array}$} \\
\hline Results & & & & & $\mathrm{Nr}$. & \\
\hline observations & 2 & 22.2 & 7 & 77.78 & 0 & 00.00 \\
\hline self-assessment & 0 & 00.0 & 1 & 10.00 & 9 & 90.00 \\
\hline $\begin{array}{c}\text { Indicator } \\
3.4 .\end{array}$ & \multicolumn{6}{|c|}{$\begin{array}{l}\text { The specialist provides } \\
\text { opportunities for children to play } \\
\text { with other children and to enjoy } \\
\text { pleasant moments together in ways } \\
\text { that encourage recognition and } \\
\text { collaboration with others. }\end{array}$} \\
\hline Statements & \multirow{2}{*}{\multicolumn{2}{|c|}{$\begin{array}{l}\text { Does not } \\
\text { apply } \\
\text { Nr. } \%\end{array}$}} & \multirow{2}{*}{\multicolumn{2}{|c|}{$\begin{array}{r}\text { Sometim } \\
\text { es apply } \\
\text { Nr. } \%\end{array}$}} & \multicolumn{2}{|c|}{$\begin{array}{l}\text { Apply } \\
\text { always }\end{array}$} \\
\hline Results & & & & & $\mathrm{Nr}$. & \\
\hline observations & 2 & 22.2 & 1 & 11.11 & 6 & 66.67 \\
\hline self-assessment & 0 & 00.0 & 0 & 00.00 & 10 & 100.00 \\
\hline $\begin{array}{c}\text { Indicator } \\
3.5 \text {. }\end{array}$ & \multicolumn{6}{|c|}{$\begin{array}{l}\text { The specialist encourages the game } \\
\text { by providing open resources that } \\
\text { support creative discovery. }\end{array}$} \\
\hline Statements & \multicolumn{2}{|c|}{$\begin{array}{c}\text { Does not } \\
\text { apply }\end{array}$} & \multicolumn{2}{|c|}{$\begin{array}{l}\text { Sometim } \\
\text { es apply }\end{array}$} & \multicolumn{2}{|c|}{$\begin{array}{l}\text { Apply } \\
\text { always }\end{array}$} \\
\hline Results & \multicolumn{2}{|c|}{$\mathrm{Nr} . \quad \%$} & \multicolumn{2}{|c|}{ Nr. $\%$} & \multicolumn{2}{|c|}{ Nr. $\quad \%$} \\
\hline observations & 2 & 22.2 & 6 & 66.67 & 1 & 11.11 \\
\hline & 0 & 00.0 & 0 & 00.00 & 10 & 100.00 \\
\hline
\end{tabular}

Table 21. Principle 4: Development and learning is promoted through support.

\begin{tabular}{|c|c|c|c|c|c|c|}
\hline $\begin{array}{c}\text { Indicator } \\
4.1 .\end{array}$ & \multicolumn{6}{|c|}{$\begin{array}{l}\text { Responding to children's signals, } \\
\text { actions and comments by providing } \\
\text { verbal and non-verbal advice, } \\
\text { assistance or questions, descriptions } \\
\text { and tips, and multi-faceted } \\
\text { communications. }\end{array}$} \\
\hline Statements & \multicolumn{2}{|c|}{$\begin{array}{c}\text { Does not } \\
\text { apply }\end{array}$} & \multicolumn{2}{|c|}{$\begin{array}{l}\text { Sometim } \\
\text { es apply }\end{array}$} & \multicolumn{2}{|c|}{$\begin{array}{l}\text { Apply } \\
\text { always }\end{array}$} \\
\hline Results & \multicolumn{2}{|c|}{ Nr. $\%$} & \multicolumn{2}{|c|}{ Nr. $\%$} & \multicolumn{2}{|c|}{ Nr. $\quad \%$} \\
\hline observations & 1 & 11.1 & 7 & 77.78 & 1 & 11.11 \\
\hline self-assessment & 0 & 00.0 & 0 & 00.00 & 10 & 100.00 \\
\hline $\begin{array}{c}\text { Indicator } \\
4.2 \text {. }\end{array}$ & \multicolumn{6}{|c|}{$\begin{array}{l}\text { Learning new concepts and skills is } \\
\text { related to the child's previous } \\
\text { knowledge and experiences. }\end{array}$} \\
\hline Statements & \multicolumn{2}{|c|}{$\begin{array}{c}\text { Does not } \\
\text { apply }\end{array}$} & \multicolumn{2}{|c|}{$\begin{array}{l}\text { Sometim } \\
\text { es apply }\end{array}$} & \multicolumn{2}{|c|}{$\begin{array}{l}\text { Apply } \\
\text { always }\end{array}$} \\
\hline Results & \multicolumn{2}{|c|}{ Nr. $\%$} & \multicolumn{2}{|c|}{ Nr. $\%$} & \multicolumn{2}{|c|}{ Nr. $\quad \%$} \\
\hline observations & 2 & 22.2 & 1 & 11.11 & 6 & 66.67 \\
\hline self-assessment & 0 & 00.0 & 0 & 00.00 & 10 & 100.00 \\
\hline $\begin{array}{c}\text { Indicator } \\
4.3 .\end{array}$ & \multicolumn{6}{|c|}{$\begin{array}{l}\text { Children are encouraged to take } \\
\text { appropriate risks to encourage their } \\
\text { development and learning by taking } \\
\text { an active part in solving their } \\
\text { problems. }\end{array}$} \\
\hline Statements & \multicolumn{2}{|c|}{$\begin{array}{c}\text { Does not } \\
\text { apply }\end{array}$} & \multicolumn{2}{|c|}{$\begin{array}{l}\text { Sometim } \\
\text { es apply }\end{array}$} & \multicolumn{2}{|c|}{$\begin{array}{l}\text { Apply } \\
\text { always }\end{array}$} \\
\hline Results & \multicolumn{2}{|c|}{ Nr. $\%$} & \multicolumn{2}{|c|}{ Nr. $\%$} & \multicolumn{2}{|c|}{ Nr. $\quad \%$} \\
\hline observations & 8 & 88.8 & 0 & 00.00 & 1 & 11.11 \\
\hline self-assessment & 0 & 00.0 & 1 & 10.00 & 9 & 90.00 \\
\hline
\end{tabular}




\subsection{Priority area "Monitoring, Documentation, Reflection and Planning"}

Table 22. Principle 1: Monitoring provides important information about child development, learning, interests, strengths and needs.

\begin{tabular}{|c|c|c|c|c|c|c|}
\hline $\begin{array}{c}\text { Indicator } \\
1.1 .\end{array}$ & \multicolumn{6}{|c|}{$\begin{array}{l}\text { The specialist values the act of } \\
\text { observing the verbal and non-verbal } \\
\text { behaviors of the child as well as the } \\
\text { context for concrete behavior as a } \\
\text { way to better understand the child } \\
\text { and motivate its decisions. }\end{array}$} \\
\hline Statements & \multirow{2}{*}{\multicolumn{2}{|c|}{$\begin{array}{l}\text { Does not } \\
\text { apply } \\
\text { Nr. } \%\end{array}$}} & \multirow{2}{*}{\multicolumn{2}{|c|}{$\begin{array}{r}\text { Sometim } \\
\text { es apply } \\
\text { Nr. } \%\end{array}$}} & \multicolumn{2}{|c|}{$\begin{array}{l}\text { Apply } \\
\text { always }\end{array}$} \\
\hline Results & & & & & & \\
\hline observations & 0 & 00.0 & 9 & 100.0 & & 00.00 \\
\hline self-assessment & 0 & 00.0 & 1 & 10.00 & & 90.00 \\
\hline $\begin{array}{c}\text { Indicator } \\
1.2 .\end{array}$ & \multicolumn{6}{|c|}{$\begin{array}{l}\text { The specialist participates in a } \\
\text { number of observations of child } \\
\text { growth, language, development and } \\
\text { learning, daily and over time. }\end{array}$} \\
\hline Statements & \multicolumn{2}{|c|}{$\begin{array}{l}\text { Does not } \\
\text { apply }\end{array}$} & \multicolumn{2}{|c|}{$\begin{array}{l}\text { Sometim } \\
\text { es apply }\end{array}$} & \multicolumn{2}{|c|}{$\begin{array}{l}\text { Apply } \\
\text { always }\end{array}$} \\
\hline Results & \multicolumn{2}{|c|}{ Nr. $\%$} & \multicolumn{2}{|c|}{ Nr. $\quad \%$} & \multicolumn{2}{|c|}{ Nr. $\quad \%$} \\
\hline observations & 0 & 00.0 & 8 & 88.89 & & 11.11 \\
\hline self-assessment & 0 & 00.0 & 1 & 10.00 & & 90.00 \\
\hline $\begin{array}{c}\text { Indicator } \\
1.3 .\end{array}$ & \multicolumn{6}{|c|}{$\begin{array}{l}\text { The specialist uses surveillance to } \\
\text { determine if changes to learning } \\
\text { practices are necessary with the } \\
\text { child. }\end{array}$} \\
\hline Statements & \multirow{2}{*}{\multicolumn{2}{|c|}{$\begin{array}{l}\text { Does not } \\
\text { apply } \\
\mathrm{Nr} \quad \%\end{array}$}} & \multicolumn{2}{|c|}{$\begin{array}{l}\text { Sometim } \\
\text { es apply }\end{array}$} & \multicolumn{2}{|c|}{$\begin{array}{c}\text { Apply } \\
\text { always }\end{array}$} \\
\hline Results & & & \multicolumn{2}{|c|}{ Nr. $\quad \%$} & \multicolumn{2}{|c|}{ Nr. $\quad \%$} \\
\hline observations & 3 & 33.3 & 1 & 11.11 & & 55.56 \\
\hline self-assessment & 0 & 00.0 & 1 & 10.00 & & 90.00 \\
\hline $\begin{array}{c}\text { Indicator } \\
1.4 .\end{array}$ & \multicolumn{6}{|c|}{$\begin{array}{l}\text { The specialist uses observation to } \\
\text { mark and document the } \\
\text { achievements of the children. }\end{array}$} \\
\hline Statements & \multicolumn{2}{|c|}{$\begin{array}{c}\text { Does not } \\
\text { apply }\end{array}$} & \multicolumn{2}{|c|}{$\begin{array}{l}\text { Sometim } \\
\text { es apply }\end{array}$} & \multicolumn{2}{|c|}{$\begin{array}{l}\text { Apply } \\
\text { always }\end{array}$} \\
\hline Results & \multicolumn{2}{|c|}{$\mathrm{Nr} . \quad \%$} & \multicolumn{2}{|c|}{ Nr. $\%$} & \multicolumn{2}{|c|}{ Nr. $\quad \%$} \\
\hline observations & 3 & 33.3 & 1 & 11.11 & & 55.56 \\
\hline self-assessment & 0 & 00.0 & 4 & 40.00 & & 60.00 \\
\hline $\begin{array}{c}\text { Indicator } \\
1.5 .\end{array}$ & \multicolumn{6}{|c|}{$\begin{array}{l}\text { Uses monitoring and screening tools } \\
\text { as a way of identifying possible } \\
\text { delays in development, disability, } \\
\text { malnutrition, chronic illness, } \\
\text { atypical behavior. }\end{array}$} \\
\hline Statements & \multirow{2}{*}{\multicolumn{2}{|c|}{$\begin{array}{l}\text { Does not } \\
\text { apply } \\
\text { Nr. } \%\end{array}$}} & \multicolumn{2}{|c|}{$\begin{array}{l}\text { Sometim } \\
\text { es apply }\end{array}$} & \multicolumn{2}{|c|}{$\begin{array}{l}\text { Apply } \\
\text { always }\end{array}$} \\
\hline Results & & & & & & \\
\hline observations & 3 & 33.3 & 6 & 66.67 & 0 & 00.00 \\
\hline self-assessment & 3 & 33.3 & 6 & 66.67 & 0 & 00.00 \\
\hline
\end{tabular}

Table 23. Principle 2: Monitoring is most useful when documenting, analyzing and sharing with parents / families and other people involved in the care and well-being of the child.

\begin{tabular}{|c|c|c|c|c|c|c|}
\hline $\begin{array}{c}\text { Indicator } \\
2.1 .\end{array}$ & \multicolumn{6}{|c|}{$\begin{array}{l}\text { The specialist records the } \\
\text { observations in a comprehensible } \\
\text { and objective way so that they can } \\
\text { be shared easily with others who } \\
\text { also work with the child (with } \\
\text { permission from the parents). }\end{array}$} \\
\hline Statements & \multirow{2}{*}{\multicolumn{2}{|c|}{$\begin{array}{l}\text { Does not } \\
\text { apply } \\
\text { Nr. } \quad \%\end{array}$}} & \multicolumn{2}{|c|}{$\begin{array}{c}\text { Sometime } \\
\text { s apply }\end{array}$} & \multicolumn{2}{|c|}{$\begin{array}{c}\text { Apply } \\
\text { always }\end{array}$} \\
\hline Results & & & & & & \\
\hline observations & 1 & 11.1 & 8 & 88.89 & 0 & 00.00 \\
\hline self-assessment & 0 & 00.0 & 3 & 30.00 & 7 & 70.00 \\
\hline $\begin{array}{c}\text { Indicator } \\
2.2 .\end{array}$ & \multicolumn{6}{|c|}{$\begin{array}{l}\text { The specialist uses various tools as } \\
\text { appropriate to record and / or } \\
\text { evaluate observations, including } \\
\text { incidental recordings, stories, } \\
\text { videos, photos, audio recordings, } \\
\text { dictations, drawings and references. }\end{array}$} \\
\hline Statements & \multicolumn{2}{|c|}{$\begin{array}{c}\text { Does not } \\
\text { apply }\end{array}$} & \multicolumn{2}{|c|}{$\begin{array}{l}\text { Sometim } \\
\text { es apply }\end{array}$} & \multicolumn{2}{|c|}{$\begin{array}{l}\text { Apply } \\
\text { always }\end{array}$} \\
\hline Results & \multicolumn{2}{|c|}{ Nr. $\%$} & \multicolumn{2}{|c|}{ Nr. $\%$} & \multicolumn{2}{|c|}{ Nr. $\%$} \\
\hline observations & 6 & 66.6 & 2 & 22.22 & 1 & 11.11 \\
\hline self-assessment & 0 & 00.0 & 4 & 40.00 & 6 & 60.00 \\
\hline $\begin{array}{c}\text { Indicator } \\
2.3 .\end{array}$ & \multicolumn{6}{|c|}{$\begin{array}{l}\text { The specialist uses a system to } \\
\text { organize monitoring and file } \\
\text { keeping so that information can be } \\
\text { used for sharing, screening, } \\
\text { evaluation, and planning purposes. }\end{array}$} \\
\hline Statements & \multirow{2}{*}{\multicolumn{2}{|c|}{$\begin{array}{l}\text { Does not } \\
\text { apply } \\
\mathrm{Nr} \quad \%\end{array}$}} & \multirow{2}{*}{\multicolumn{2}{|c|}{$\begin{array}{r}\text { Sometim } \\
\text { es apply } \\
\mathrm{Nr} \quad \%\end{array}$}} & \multirow{2}{*}{\multicolumn{2}{|c|}{$\begin{array}{c}\text { Apply } \\
\text { always } \\
\% \quad \%\end{array}$}} \\
\hline Results & & & & & & \\
\hline observations & 7 & 77.7 & 2 & 22.22 & 0 & 00.00 \\
\hline self-assessment & 0 & 00.0 & 4 & 40.00 & 6 & 60.00 \\
\hline $\begin{array}{c}\text { Indicator } \\
2.4 .\end{array}$ & \multicolumn{6}{|c|}{$\begin{array}{l}\text { The specialist shares observations } \\
\text { with families and other } \\
\text { professionals (when parental } \\
\text { permission is granted) working with } \\
\text { the child to ensure seamless care for } \\
\text { the child and engaging in any } \\
\text { interference and involvement of } \\
\text { other services. }\end{array}$} \\
\hline Statements & \multicolumn{2}{|c|}{$\begin{array}{c}\text { Does not } \\
\text { apply }\end{array}$} & \multicolumn{2}{|c|}{$\begin{array}{l}\text { Sometim } \\
\text { es apply }\end{array}$} & \multicolumn{2}{|c|}{$\begin{array}{l}\text { Apply } \\
\text { always }\end{array}$} \\
\hline Results & \multicolumn{2}{|c|}{$\mathrm{Nr} . \%$} & \multicolumn{2}{|c|}{$\mathrm{Nr} . \quad \%$} & \multicolumn{2}{|c|}{ Nr. $\quad \%$} \\
\hline observations & 2 & 22.2 & 7 & 77.78 & 0 & 00.00 \\
\hline self-assessment & 0 & 00.0 & 2 & 20.00 & 8 & 80.00 \\
\hline $\begin{array}{c}\text { Indicator } \\
2.5 .\end{array}$ & \multicolumn{6}{|c|}{$\begin{array}{l}\text { The specialist specifies specific } \\
\text { hours for reviewing the } \\
\text { documentation with other key adults } \\
\text { involved in child care. }\end{array}$} \\
\hline Statements & \multirow{2}{*}{\multicolumn{2}{|c|}{$\begin{array}{l}\text { Does not } \\
\text { apply }\end{array}$}} & \multicolumn{2}{|c|}{$\begin{array}{l}\text { Sometim } \\
\text { es apply }\end{array}$} & \multicolumn{2}{|c|}{$\begin{array}{l}\text { Apply } \\
\text { always }\end{array}$} \\
\hline Results & & & & & & \\
\hline observations & 8 & 88.8 & 1 & 11.11 & 0 & 00.00 \\
\hline self-assessment & 4 & 40.0 & 2 & 20.00 & 4 & 40.00 \\
\hline
\end{tabular}


Table 24. Principle 3: The joint discussion of children's observations and the documentation of their learning and socialization experiences motivates the review of professional practices and their future improvement.

\begin{tabular}{|c|c|c|c|c|c|c|}
\hline $\begin{array}{c}\text { Indicator } \\
2.1\end{array}$ & \multicolumn{6}{|c|}{$\begin{array}{l}\text { The specialist records the } \\
\text { observations in a comprehensible } \\
\text { and objective way so that they can } \\
\text { be shared easily with others who } \\
\text { also work with the child (with } \\
\text { permission from the parents). }\end{array}$} \\
\hline Statements & \multirow{2}{*}{\multicolumn{2}{|c|}{$\begin{array}{l}\text { Does not } \\
\text { apply } \\
\text { Nr. } \%\end{array}$}} & \multirow{2}{*}{\multicolumn{2}{|c|}{ Sometime }} & \multicolumn{2}{|c|}{$\begin{array}{l}\text { Apply } \\
\text { always }\end{array}$} \\
\hline Results & & & & & & \\
\hline observations & 1 & 11.1 & 8 & 88.89 & 0 & 00.00 \\
\hline self-assessment & 0 & 00.0 & 3 & 30.00 & 7 & 70.00 \\
\hline $\begin{array}{c}\text { Indicator } \\
2.2\end{array}$ & \multicolumn{6}{|c|}{$\begin{array}{l}\text { The specialist uses various tools as } \\
\text { appropriate to record and / or } \\
\text { evaluate observations, including } \\
\text { incidental recordings, stories, } \\
\text { videos, photos, audio recordings, } \\
\text { dictations, drawings and references. }\end{array}$} \\
\hline Statements & \multicolumn{2}{|c|}{$\begin{array}{c}\text { Does not } \\
\text { apply }\end{array}$} & \multicolumn{2}{|c|}{$\begin{array}{l}\text { Sometim } \\
\text { es apply }\end{array}$} & \multicolumn{2}{|c|}{$\begin{array}{l}\text { Apply } \\
\text { always }\end{array}$} \\
\hline Results & \multicolumn{2}{|c|}{ Nr. \% } & \multicolumn{2}{|c|}{ Nr. $\%$} & \multicolumn{2}{|c|}{ Nr. $\quad \%$} \\
\hline observations & 6 & 66.6 & 2 & 22.22 & 1 & 11.11 \\
\hline self-assessment & 0 & 00.0 & 4 & 40.00 & 6 & 60.00 \\
\hline $\begin{array}{c}\text { Indicator } \\
2.3\end{array}$ & \multicolumn{6}{|c|}{$\begin{array}{l}\text { The specialist uses a system to } \\
\text { organize monitoring and file } \\
\text { keeping so that information can be } \\
\text { used for sharing, screening, } \\
\text { evaluation, and planning purposes. }\end{array}$} \\
\hline Statements & \multicolumn{2}{|c|}{$\begin{array}{c}\text { Does not } \\
\text { apply }\end{array}$} & \multicolumn{2}{|c|}{$\begin{array}{l}\text { Sometim } \\
\text { es apply }\end{array}$} & \multicolumn{2}{|c|}{$\begin{array}{l}\text { Apply } \\
\text { always }\end{array}$} \\
\hline Results & \multicolumn{2}{|c|}{$\mathrm{Nr} . \%$} & \multicolumn{2}{|c|}{ Nr. $\%$} & \multicolumn{2}{|c|}{ Nr. $\%$} \\
\hline observations & 7 & 77.7 & 2 & 22.22 & 0 & 00.00 \\
\hline self-assessment & 0 & 00.0 & 4 & 40.00 & 6 & 60.00 \\
\hline $\begin{array}{c}\text { Indicator } \\
2.4 \text {. }\end{array}$ & \multicolumn{6}{|c|}{$\begin{array}{l}\text { The specialist shares observations } \\
\text { with families and other } \\
\text { professionals (when parental } \\
\text { permission is granted) working with } \\
\text { the child to ensure seamless care for } \\
\text { the child and engaging in any } \\
\text { interference and involvement of } \\
\text { other services. }\end{array}$} \\
\hline Statements & \multirow{2}{*}{\multicolumn{2}{|c|}{$\begin{array}{c}\text { Does not } \\
\text { apply }\end{array}$}} & \multicolumn{2}{|c|}{$\begin{array}{l}\text { Sometim } \\
\text { es apply }\end{array}$} & \multicolumn{2}{|c|}{$\begin{array}{l}\text { Apply } \\
\text { always }\end{array}$} \\
\hline Results & $\mathrm{Nr} . \%$ & & \multicolumn{2}{|c|}{ Nr. $\%$} & \multicolumn{2}{|c|}{ Nr. $\%$} \\
\hline observations & 2 & 22.2 & 7 & 77.78 & 0 & 00.00 \\
\hline self-assessment & 0 & 00.0 & & 20.00 & 8 & 80.00 \\
\hline $\begin{array}{c}\text { Indicator } \\
2.5 .\end{array}$ & \multicolumn{6}{|c|}{$\begin{array}{l}\text { The specialist specifies specific } \\
\text { hours for reviewing the } \\
\text { documentation with other key adults } \\
\text { involved in child care. }\end{array}$} \\
\hline Statements & \multirow{2}{*}{\multicolumn{2}{|c|}{$\begin{array}{c}\text { Does not } \\
\text { apply } \\
\mathrm{Nr} \quad \%\end{array}$}} & \multicolumn{2}{|c|}{$\begin{array}{l}\text { Sometim } \\
\text { es apply }\end{array}$} & \multicolumn{2}{|c|}{$\begin{array}{l}\text { Apply } \\
\text { always }\end{array}$} \\
\hline Results & & & & & & \\
\hline observations & 8 & 88.8 & 1 & 11.11 & 0 & 00.00 \\
\hline self-assessmer & 4 & 40.0 & 2 & 20.00 & 4 & 40.00 \\
\hline
\end{tabular}

Table 25. Principle 4: Long-term and short-term planning is based on individual children's strengths and needs.

\begin{tabular}{|c|c|c|c|c|c|c|}
\hline $\begin{array}{c}\text { Indicator } \\
4.1 .\end{array}$ & \multicolumn{6}{|c|}{$\begin{array}{l}\text { Observations and documentation are } \\
\text { used to develop long-term plans for } \\
\text { child learning, including any } \\
\text { changes that need to be made to the } \\
\text { interactions of the adult with the } \\
\text { child and the environment. }\end{array}$} \\
\hline Statements & \multirow{2}{*}{\multicolumn{2}{|c|}{$\begin{array}{l}\text { Does not } \\
\text { apply } \\
\mathrm{Nr} \quad \%\end{array}$}} & \multicolumn{2}{|c|}{$\begin{array}{l}\text { Sometim } \\
\text { es apply }\end{array}$} & \multicolumn{2}{|c|}{$\begin{array}{c}\text { Apply } \\
\text { always }\end{array}$} \\
\hline Results & & & & & & \\
\hline observations & 7 & 77.7 & 2 & 22.22 & 0 & 00.00 \\
\hline self-asses & 0 & 00.0 & 1 & 10.00 & 9 & 90.00 \\
\hline $\begin{array}{c}\text { Indicator } \\
4.2\end{array}$ & \multicolumn{6}{|c|}{$\begin{array}{l}\text { Plans are created for the } \\
\text { opportunities for future learning } \\
\text { activities based on the responses, } \\
\text { interests, strengths and needs of } \\
\text { each child. }\end{array}$} \\
\hline Statements & \multicolumn{2}{|c|}{$\begin{array}{c}\text { Does not } \\
\text { apply }\end{array}$} & \multicolumn{2}{|c|}{$\begin{array}{l}\text { Sometim } \\
\text { es apply }\end{array}$} & \multicolumn{2}{|c|}{$\begin{array}{l}\text { Apply } \\
\text { always }\end{array}$} \\
\hline Results & \multicolumn{2}{|c|}{ Nr. $\%$} & \multicolumn{2}{|c|}{ Nr. $\%$} & \multicolumn{2}{|c|}{ Nr. $\quad \%$} \\
\hline observations & 8 & 88.8 & 1 & 11.11 & 0 & 00.00 \\
\hline self-assessment & 0 & 00.0 & 9 & 90.00 & 1 & 10.00 \\
\hline $\begin{array}{c}\text { Indicator } \\
4.3 .\end{array}$ & \multicolumn{6}{|c|}{$\begin{array}{l}\text { Plans are followed, following each } \\
\text { child's management, as it interacts } \\
\text { with materials, people and nature in } \\
\text { the surrounding environment, } \\
\text { making changes when and when it } \\
\text { is needed. }\end{array}$} \\
\hline Statements & \multirow{2}{*}{\multicolumn{2}{|c|}{$\begin{array}{l}\text { Does not } \\
\text { apply } \\
\text { Nr. } \%\end{array}$}} & \multicolumn{2}{|c|}{$\begin{array}{l}\text { Sometim } \\
\text { es apply }\end{array}$} & \multicolumn{2}{|c|}{$\begin{array}{l}\text { Apply } \\
\text { always }\end{array}$} \\
\hline Results & & & & & & \\
\hline observations & 1 & 11.1 & 8 & 88.89 & 0 & 00.00 \\
\hline self-assessment & 0 & 00.0 & 6 & 60.00 & 4 & 40.00 \\
\hline
\end{tabular}




\subsection{Priority area "Supporting Environments "}

Table 26. Principle 1: The environment encourages the emotional well-being of each child.

\begin{tabular}{|c|c|c|c|c|c|c|}
\hline $\begin{array}{c}\text { Indicator } \\
1.1 .\end{array}$ & \multicolumn{6}{|c|}{$\begin{array}{l}\text { The specialist demonstrates } \\
\text { familiarity with stressors in the } \\
\text { surrounding environment and strives } \\
\text { to reduce them. }\end{array}$} \\
\hline Statements & \multicolumn{2}{|c|}{$\begin{array}{c}\text { Does not } \\
\text { apply }\end{array}$} & \multicolumn{2}{|c|}{$\begin{array}{l}\text { Sometim } \\
\text { es apply }\end{array}$} & \multicolumn{2}{|c|}{$\begin{array}{l}\text { Apply } \\
\text { always }\end{array}$} \\
\hline Results & \multicolumn{2}{|c|}{$\mathrm{Nr} . \quad \%$} & \multicolumn{2}{|c|}{ Nr. $\%$} & \multicolumn{2}{|c|}{ Nr. $\quad \%$} \\
\hline observations & 0 & 00.0 & 3 & 33.33 & 6 & 66.67 \\
\hline self-as & 0 & 00.0 & 1 & 10.00 & 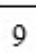 & 90.00 \\
\hline $\begin{array}{l}\text { Indicator } \\
1.2 .\end{array}$ & \multicolumn{6}{|c|}{$\begin{array}{l}\text { The specialist provides a place for } \\
\text { rest, sleep and relaxation. }\end{array}$} \\
\hline Statements & \multicolumn{2}{|c|}{$\begin{array}{l}\text { Does not } \\
\text { apply }\end{array}$} & \multicolumn{2}{|c|}{$\begin{array}{l}\text { Sometim } \\
\text { es apply }\end{array}$} & \multicolumn{2}{|c|}{$\begin{array}{l}\text { Apply } \\
\text { always }\end{array}$} \\
\hline Results & \multicolumn{2}{|c|}{$\mathrm{Nr} . \quad \%$} & \multicolumn{2}{|c|}{ Nr. $\%$} & \multicolumn{2}{|c|}{ Nr. $\quad \%$} \\
\hline observations & 0 & 00.0 & 2 & 22.22 & 8 & 88.89 \\
\hline self-asse & 0 & 00.0 & 1 & 10.00 & 9 & 90.00 \\
\hline $\begin{array}{c}\text { Indicator } \\
1.3 .\end{array}$ & \multicolumn{6}{|c|}{$\begin{array}{l}\text { The specialist has expectations and } \\
\text { directs the behavior of the child in a } \\
\text { way that matches and is appropriate } \\
\text { for the age of the child. }\end{array}$} \\
\hline Statements & \multicolumn{2}{|c|}{$\begin{array}{l}\text { Does not } \\
\text { apply }\end{array}$} & \multicolumn{2}{|c|}{$\begin{array}{l}\text { Sometim } \\
\text { es apply }\end{array}$} & \multicolumn{2}{|c|}{$\begin{array}{l}\text { Apply } \\
\text { always }\end{array}$} \\
\hline Results & \multicolumn{2}{|c|}{$\mathrm{Nr} . \%$} & \multicolumn{2}{|c|}{ Nr. $\%$} & \multicolumn{2}{|c|}{ Nr. $\quad \%$} \\
\hline observations & 2 & 22.2 & 1 & 11.11 & 0 & 66.67 \\
\hline $\begin{array}{c}\text { Indicator } \\
1.4 .\end{array}$ & \multicolumn{6}{|c|}{$\begin{array}{l}\text { The specialist maintains a physical } \\
\text { and emotional environment that } \\
\text { minimizes conflicts and fosters } \\
\text { positive interactions among young } \\
\text { children by providing sufficient } \\
\text { resources and positive interference. }\end{array}$} \\
\hline Statements & \multicolumn{2}{|c|}{$\begin{array}{c}\text { Does not } \\
\text { apply }\end{array}$} & \multicolumn{2}{|c|}{$\begin{array}{l}\text { Sometim } \\
\text { es apply }\end{array}$} & \multicolumn{2}{|c|}{$\begin{array}{l}\text { Apply } \\
\text { always }\end{array}$} \\
\hline Results & $\mathrm{Nr}$ & $\%$ & $\mathrm{Nr}$ & & & \\
\hline observations & 2 & 22.2 & 1 & 11.11 & 6 & 66.67 \\
\hline self-a: & 0 & 00.0 & 1 & 10.00 & 9 & 90.00 \\
\hline
\end{tabular}

Table 27. Principle 2: The environment is safe, clean and free from all contamination and controlled.

\begin{tabular}{|c|c|c|c|c|c|c|}
\hline $\begin{array}{c}\text { Indicator } \\
2.1 .\end{array}$ & \multicolumn{6}{|c|}{$\begin{array}{l}\text { The specialist provides and prevents } \\
\text { safety problems. }\end{array}$} \\
\hline Statements & \multirow{2}{*}{\multicolumn{2}{|c|}{$\begin{array}{l}\text { Does not } \\
\text { apply } \\
\text { \%r } \%\end{array}$}} & \multirow{2}{*}{\multicolumn{2}{|c|}{$\begin{array}{l}\text { Sometim } \\
\text { es apply } \\
\mathrm{Nr} \%\end{array}$}} & \multicolumn{2}{|c|}{$\begin{array}{l}\text { Apply } \\
\text { always }\end{array}$} \\
\hline Results & & & & & $\mathrm{Nr}$. & $\%$ \\
\hline observations & 1 & 11.1 & 1 & 11.11 & 7 & 77.78 \\
\hline self-asse & 0 & 00.0 & 1 & 10.00 & 9 & 90.00 \\
\hline $\begin{array}{c}\text { Indicator } \\
2.2\end{array}$ & \multicolumn{6}{|c|}{$\begin{array}{l}\text { The specialist ensures that the } \\
\text { environment is clean, hygienic and } \\
\text { free from dirt. }\end{array}$} \\
\hline Statements & \multicolumn{2}{|c|}{$\begin{array}{l}\text { Does not } \\
\text { apply }\end{array}$} & \multicolumn{2}{|c|}{$\begin{array}{l}\text { Sometim } \\
\text { es apply }\end{array}$} & \multicolumn{2}{|c|}{$\begin{array}{l}\text { Apply } \\
\text { always }\end{array}$} \\
\hline Results & \multicolumn{2}{|c|}{$\mathrm{Nr} . \quad \%$} & \multicolumn{2}{|c|}{ Nr. $\%$} & \multicolumn{2}{|c|}{ Nr. $\quad \%$} \\
\hline observations & 1 & 11.1 & 1 & 11.11 & 7 & 77.78 \\
\hline self-assessi & 0 & 00.0 & 0 & 00.00 & 10 & 100.0 \\
\hline $\begin{array}{c}\text { Indicator } \\
2.3\end{array}$ & \multicolumn{6}{|c|}{$\begin{array}{l}\text { The specialist monitors the good } \\
\text { condition of the materials and } \\
\text { furniture. }\end{array}$} \\
\hline Statements & \multirow{2}{*}{\multicolumn{2}{|c|}{$\begin{array}{l}\text { Does not } \\
\text { apply } \\
\text { Nr. } \%\end{array}$}} & \multicolumn{2}{|c|}{$\begin{array}{l}\text { Sometim } \\
\text { es apply }\end{array}$} & \multicolumn{2}{|c|}{$\begin{array}{l}\text { Apply } \\
\text { always }\end{array}$} \\
\hline Results & & & \multicolumn{2}{|c|}{ Nr. $\%$} & \multicolumn{2}{|c|}{ Nr. $\quad \%$} \\
\hline observations & 1 & 11.1 & 2 & 22.22 & 6 & 66.67 \\
\hline self-assessm & 0 & 00.0 & 0 & 00.00 & 10 & 100.0 \\
\hline $\begin{array}{c}\text { Indicator } \\
2.4\end{array}$ & \multicolumn{6}{|c|}{$\begin{array}{l}\text { The specialist is convinced that } \\
\text { children are under constant } \\
\text { observation, even when they are } \\
\text { sleeping. }\end{array}$} \\
\hline Statements & \multicolumn{2}{|c|}{$\begin{array}{c}\text { Does not } \\
\text { apply }\end{array}$} & \multicolumn{2}{|c|}{$\begin{array}{l}\text { Sometim } \\
\text { es apply }\end{array}$} & \multicolumn{2}{|c|}{$\begin{array}{l}\text { Apply } \\
\text { always }\end{array}$} \\
\hline Results & $\mathrm{Nr}$ & $\%$ & & & $\mathrm{Nr}$. & \\
\hline rvations & 0 & 00.0 & 0 & 00.00 & 9 & 100.0 \\
\hline self-as & 0 & 00.0 & 0 & 00.00 & 10 & 100.0 \\
\hline
\end{tabular}


Table 28. Principle 3: The environment is hospitable, accessible and comfortable.

\begin{tabular}{|c|c|c|c|c|c|c|}
\hline $\begin{array}{c}\text { Indicator } \\
3.1 .\end{array}$ & \multicolumn{6}{|c|}{$\begin{array}{l}\text { The specialist arranges space for } \\
\text { children to respond to their current } \\
\text { needs, interests and the ability to } \\
\text { move freely. }\end{array}$} \\
\hline Statements & \multicolumn{2}{|c|}{$\begin{array}{c}\text { Does not } \\
\text { apply }\end{array}$} & \multicolumn{2}{|c|}{$\begin{array}{l}\text { Sometim } \\
\text { es apply }\end{array}$} & \multicolumn{2}{|c|}{$\begin{array}{c}\text { Apply } \\
\text { always }\end{array}$} \\
\hline Results & \multicolumn{2}{|c|}{ Nr. $\%$} & \multicolumn{2}{|c|}{ Nr. $\%$} & \multicolumn{2}{|c|}{ Nr. $\%$} \\
\hline observations & 1 & 11.1 & 2 & 22.22 & 6 & 66.67 \\
\hline self-as & 0 & 00.0 & 1 & 10.00 & & 90.00 \\
\hline $\begin{array}{c}\text { Indicator } \\
3.2 \text {. }\end{array}$ & \multicolumn{6}{|c|}{$\begin{array}{l}\text { The specialist is convinced that the } \\
\text { equipment and materials areas } \\
\text { required for care are conveniently } \\
\text { located. }\end{array}$} \\
\hline Statements & \multicolumn{2}{|c|}{$\begin{array}{c}\text { Does not } \\
\text { apply }\end{array}$} & \multicolumn{2}{|c|}{$\begin{array}{l}\text { Sometim } \\
\text { es apply }\end{array}$} & \multicolumn{2}{|c|}{$\begin{array}{c}\text { Apply } \\
\text { always }\end{array}$} \\
\hline Results & \multicolumn{2}{|c|}{ Nr. $\%$} & \multicolumn{2}{|c|}{ Nr. $\%$} & \multicolumn{2}{|c|}{ Nr. $\%$} \\
\hline observations & 2 & 22.2 & 1 & 11.11 & 6 & 66.67 \\
\hline self-asse & 0 & 00.0 & 1 & 10.00 & s & 90.00 \\
\hline $\begin{array}{c}\text { Indicator } \\
3.3 .\end{array}$ & \multicolumn{6}{|c|}{$\begin{array}{l}\text { The specialist arranges space so that } \\
\text { family members feel comfortable } \\
\text { and well during all inspections. }\end{array}$} \\
\hline Statements & \multicolumn{2}{|c|}{$\begin{array}{c}\text { Does not } \\
\text { apply }\end{array}$} & \multirow{2}{*}{\multicolumn{2}{|c|}{$\begin{array}{l}\text { Sometim } \\
\text { es apply } \\
\mathrm{Nr} \quad \%\end{array}$}} & \multicolumn{2}{|c|}{$\begin{array}{l}\text { Apply } \\
\text { always }\end{array}$} \\
\hline Results & \multicolumn{2}{|c|}{ Nr. $\%$} & & & \multicolumn{2}{|c|}{ Nr. $\%$} \\
\hline observations & 2 & 22.2 & 1 & 11.11 & & 66.67 \\
\hline self-assessment & 0 & 00.0 & 1 & 10.00 & 9 & 90.00 \\
\hline $\begin{array}{c}\text { Indicator } \\
3.4 \text {. }\end{array}$ & \multicolumn{6}{|c|}{$\begin{array}{l}\text { The specialist provides spaces that } \\
\text { are warm, soft and predispose to } \\
\text { proximity. }\end{array}$} \\
\hline Statements & \multicolumn{2}{|c|}{$\begin{array}{c}\text { Does not } \\
\text { apply }\end{array}$} & \multicolumn{2}{|c|}{$\begin{array}{l}\text { Sometim } \\
\text { es apply }\end{array}$} & \multicolumn{2}{|c|}{$\begin{array}{l}\text { Apply } \\
\text { always }\end{array}$} \\
\hline Results & \multicolumn{2}{|c|}{ Nr. $\%$} & \multicolumn{2}{|c|}{ Nr. $\%$} & \multicolumn{2}{|c|}{ Nr. $\%$} \\
\hline observations & 2 & 22.2 & 1 & 11.11 & 0 & 66.67 \\
\hline self-as & 2 & 22.2 & 1 & 11.11 & 6 & 66.67 \\
\hline
\end{tabular}

Table 29. Principle 4: The environment stimulates children's play, discovery, independence and initiative.

\begin{tabular}{|c|c|c|c|c|c|c|}
\hline $\begin{array}{l}\text { Indicator } \\
\quad 4.1 .\end{array}$ & \multicolumn{6}{|c|}{$\begin{array}{l}\text { The environment is organized in a } \\
\text { way that supports game, discovery, } \\
\text { autonomy and leadership } \\
\text { (decision-making). }\end{array}$} \\
\hline Statements & \multicolumn{2}{|c|}{$\begin{array}{l}\text { Does not } \\
\text { apply }\end{array}$} & \multicolumn{2}{|c|}{$\begin{array}{l}\text { Sometim } \\
\text { es apply }\end{array}$} & \multicolumn{2}{|c|}{$\begin{array}{l}\text { Apply } \\
\text { always }\end{array}$} \\
\hline Results & \multicolumn{2}{|c|}{ Nr. $\%$} & \multicolumn{2}{|c|}{ Nr. $\%$} & \multicolumn{2}{|c|}{ Nr. $\%$} \\
\hline observations & 0 & 00.0 & 1 & 11.11 & 8 & 88.89 \\
\hline self-assessment & 0 & 00.0 & 1 & 10.00 & 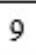 & 90.00 \\
\hline $\begin{array}{c}\text { Indicator } \\
4.2 .\end{array}$ & \multicolumn{6}{|c|}{$\begin{array}{l}\text { Game materials are provided to } \\
\text { stimulate all areas of development. }\end{array}$} \\
\hline Statements & \multicolumn{2}{|c|}{$\begin{array}{l}\text { Does not } \\
\text { apply }\end{array}$} & \multicolumn{2}{|c|}{$\begin{array}{l}\text { Sometim } \\
\text { es apply }\end{array}$} & \multicolumn{2}{|c|}{$\begin{array}{l}\text { Apply } \\
\text { always }\end{array}$} \\
\hline Results & \multicolumn{2}{|c|}{ Nr. $\%$} & \multicolumn{2}{|c|}{ Nr. $\%$} & \multicolumn{2}{|c|}{ Nr. $\%$} \\
\hline observations & 1 & 11.1 & 2 & 22.22 & 6 & 66.67 \\
\hline self-assessment & 0 & 00.0 & 1 & 10.00 & 9 & 90.00 \\
\hline $\begin{array}{l}\text { Indicator } \\
4.3 \text {. }\end{array}$ & \multicolumn{6}{|c|}{$\begin{array}{l}\text { A variety of open-source materials } \\
\text { are provided to combine to stimulate } \\
\text { gameplay and discovery. }\end{array}$} \\
\hline Statements & \multirow{2}{*}{\multicolumn{2}{|c|}{$\begin{array}{l}\text { Does not } \\
\text { apply } \\
\text { Nr. } \%\end{array}$}} & \multicolumn{2}{|c|}{$\begin{array}{l}\text { Sometim } \\
\text { es apply }\end{array}$} & \multicolumn{2}{|c|}{$\begin{array}{l}\text { Apply } \\
\text { always }\end{array}$} \\
\hline Results & & & \multicolumn{2}{|c|}{ Nr. $\%$} & \multicolumn{2}{|c|}{ Nr. $\%$} \\
\hline observations & 3 & 33.3 & 0 & 00.00 & 6 & 66.67 \\
\hline self-as & 0 & 00.0 & 1 & 10.00 & 9 & 90.00 \\
\hline $\begin{array}{c}\text { Indicator } \\
4.4 .\end{array}$ & \multicolumn{6}{|c|}{$\begin{array}{l}\text { Children are provided to open } \\
\text { spaces for at least part of the day. }\end{array}$} \\
\hline Statements & \multirow{2}{*}{\multicolumn{2}{|c|}{$\begin{array}{l}\text { Does not } \\
\text { apply } \\
\text { Nr. } \%\end{array}$}} & \multicolumn{2}{|c|}{$\begin{array}{l}\text { Sometim } \\
\text { es apply }\end{array}$} & \multicolumn{2}{|c|}{$\begin{array}{c}\text { Apply } \\
\text { always }\end{array}$} \\
\hline Results & & & \multicolumn{2}{|c|}{ Nr. $\%$} & \multicolumn{2}{|c|}{ Nr. $\%$} \\
\hline observations & 2 & 22.2 & 1 & 11.11 & 6 & 66.67 \\
\hline self-assessment & 0 & 00.0 & 2 & 20.00 & 8 & 80.00 \\
\hline $\begin{array}{c}\text { Indicator } \\
4.5 \text {. }\end{array}$ & \multicolumn{6}{|c|}{$\begin{array}{l}\text { Providing an environment that } \\
\text { encourages children to be active } \\
\text { participants and take appropriate } \\
\text { risks. }\end{array}$} \\
\hline Statements & \multirow{2}{*}{\multicolumn{2}{|c|}{$\begin{array}{l}\text { Does not } \\
\text { apply } \\
\text { Nr. } \%\end{array}$}} & \multicolumn{2}{|c|}{$\begin{array}{l}\text { Sometim } \\
\text { es apply }\end{array}$} & \multicolumn{2}{|c|}{$\begin{array}{l}\text { Apply } \\
\text { always }\end{array}$} \\
\hline Results & & & \multicolumn{2}{|c|}{ Nr. $\%$} & & \\
\hline observations & 3 & 33.3 & 0 & 00.00 & 0 & 66.67 \\
\hline self-assessment & 0 & 00.0 & 1 & 10.00 & 9 & 90.00 \\
\hline $\begin{array}{l}\text { Indicator } \\
\quad 4.6 .\end{array}$ & & $\begin{array}{l}\text { speci } \\
\text { it of } \\
\text { for } \mathrm{c} \\
\text { ng pa } \\
\text { fende }\end{array}$ & & $\begin{array}{l}\text { active } \\
\text { roup } \\
\text { but pr } \\
\text { childr }\end{array}$ & & $\begin{array}{l}\text { icipates } \\
\text { urce of } \\
\text { ed as a } \\
\text { enough } \\
0 \text { be }\end{array}$ \\
\hline Statements & & ply & & $\begin{array}{l}\text { metim } \\
\text { apply }\end{array}$ & & $\begin{array}{l}\text { pply } \\
\text { ways }\end{array}$ \\
\hline Results & $\mathrm{Nr}$ & $\%$ & & & & \\
\hline observations & 0 & 00.0 & 2 & 22.22 & 7 & 77.78 \\
\hline self-ass & 0 & 00.0 & 1 & 10.00 & 9 & 90.00 \\
\hline
\end{tabular}


Table 30. Principle 5: The environment encourages each child's sense of belonging.

\begin{tabular}{|c|c|c|c|c|c|c|}
\hline $\begin{array}{c}\text { Indicator } \\
5.1 .\end{array}$ & \multicolumn{6}{|c|}{$\begin{array}{l}\text { The specialist shows pictures of } \\
\text { children and their home } \\
\text { environment that show that the child } \\
\text { belongs to the surrounding } \\
\text { environment. }\end{array}$} \\
\hline Statements & \multicolumn{2}{|c|}{$\begin{array}{c}\text { Does not } \\
\text { apply }\end{array}$} & \multicolumn{2}{|c|}{$\begin{array}{l}\text { Sometim } \\
\text { es apply }\end{array}$} & \multicolumn{2}{|c|}{$\begin{array}{c}\text { Apply } \\
\text { always }\end{array}$} \\
\hline Results & \multicolumn{2}{|c|}{ Nr. $\%$} & \multicolumn{2}{|c|}{ Nr. $\%$} & \multicolumn{2}{|c|}{ Nr. $\%$} \\
\hline observations & 2 & 22.2 & 6 & 66.67 & 1 & 11.11 \\
\hline self-asses: & 8 & 80.0 & 0 & 00.00 & 2 & 20.00 \\
\hline $\begin{array}{c}\text { Indicator } \\
5.2 \text {. }\end{array}$ & \multicolumn{6}{|c|}{$\begin{array}{l}\text { The specialist shows respect for all } \\
\text { childhood experiences and } \\
\text { self-expression. }\end{array}$} \\
\hline Statements & \multicolumn{2}{|c|}{$\begin{array}{c}\text { Does not } \\
\text { apply }\end{array}$} & \multicolumn{2}{|c|}{$\begin{array}{l}\text { Sometim } \\
\text { es apply }\end{array}$} & \multicolumn{2}{|c|}{$\begin{array}{l}\text { Apply } \\
\text { always }\end{array}$} \\
\hline Results & \multicolumn{2}{|c|}{ Nr. $\%$} & \multicolumn{2}{|c|}{ Nr. $\%$} & \multicolumn{2}{|c|}{ Nr. $\%$} \\
\hline observations & 1 & 11.1 & 1 & 11.11 & 8 & 88.89 \\
\hline self-assess & 0 & 00.0 & 1 & 10.00 & 7 & 90.00 \\
\hline $\begin{array}{c}\text { Indicator } \\
5.3 \text {. }\end{array}$ & \multicolumn{6}{|c|}{$\begin{array}{l}\text { The specialist provides materials } \\
\text { and resources from the local } \\
\text { community and culture as well as } \\
\text { from nature. }\end{array}$} \\
\hline Statements & \multicolumn{2}{|c|}{$\begin{array}{c}\text { Does not } \\
\text { apply }\end{array}$} & \multicolumn{2}{|c|}{$\begin{array}{l}\text { Sometim } \\
\text { es apply }\end{array}$} & \multicolumn{2}{|c|}{$\begin{array}{l}\text { Apply } \\
\text { always }\end{array}$} \\
\hline Results & \multicolumn{2}{|c|}{$\mathrm{Nr} . \%$} & \multicolumn{2}{|c|}{ Nr. $\%$} & \multicolumn{2}{|c|}{ Nr. $\%$} \\
\hline observations & 3 & 33.3 & 0 & 00.00 & -1 & 66.67 \\
\hline self-assesst & 0 & 00.0 & 1 & 10.00 & $y$ & 90.00 \\
\hline
\end{tabular}

\subsection{Priority area "Professional Development"}

Table 31. Principle 1: Knowledge of children's development and learning contributes to the quality of early childhood experiences.

\begin{tabular}{|c|c|c|c|c|c|c|}
\hline $\begin{array}{c}\text { Indicator } \\
1.1 .\end{array}$ & \multicolumn{6}{|c|}{$\begin{array}{l}\text { The specialist achieves competence } \\
\text { and ability to interpret basic theories } \\
\text { of development, neurology and } \\
\text { education as a complement to } \\
\text { related research results. }\end{array}$} \\
\hline Statements & \multirow{2}{*}{\multicolumn{2}{|c|}{$\begin{array}{l}\text { Does not } \\
\text { apply } \\
\text { Nr. } \%\end{array}$}} & \multicolumn{2}{|c|}{ Sometim } & \multicolumn{2}{|c|}{$\begin{array}{l}\text { Apply } \\
\text { always }\end{array}$} \\
\hline Results & & & & $\%$ & $\mathrm{~N}$ & \\
\hline observations & 0 & 00.0 & 4 & 44.44 & 5 & 55.56 \\
\hline self-assessment & 0 & 00.0 & 8 & 80.00 & 2 & 20.00 \\
\hline $\begin{array}{c}\text { Indicator } \\
1.2 .\end{array}$ & \multicolumn{6}{|c|}{$\begin{array}{l}\text { The specialist has a holistic view of } \\
\text { development and learning about the } \\
\text { child's overall experiences. }\end{array}$} \\
\hline Statements & \multirow{2}{*}{\multicolumn{2}{|c|}{$\begin{array}{l}\text { Does not } \\
\text { apply } \\
\mathrm{Nr} . \%\end{array}$}} & \multicolumn{2}{|c|}{$\begin{array}{l}\text { Sometim } \\
\text { es apply }\end{array}$} & \multicolumn{2}{|c|}{$\begin{array}{l}\text { Apply } \\
\text { always }\end{array}$} \\
\hline Results & & & \multicolumn{2}{|c|}{ Nr. $\%$} & \multicolumn{2}{|c|}{ Nr. $\quad \%$} \\
\hline observations & 0 & 00.0 & 4 & 44.44 & 5 & 55.56 \\
\hline self-assessment & 0 & 00.0 & 1 & 10.00 & 9 & 90.00 \\
\hline $\begin{array}{c}\text { Indicator } \\
1.3 .\end{array}$ & \multicolumn{6}{|c|}{$\begin{array}{l}\text { The specialist identifies the main } \\
\text { areas of experience in the child's } \\
\text { holistic development } \\
\text { communicate sensitively with the } \\
\text { family. }\end{array}$} \\
\hline Statements & \multirow{2}{*}{\multicolumn{2}{|c|}{$\begin{array}{l}\text { Does not } \\
\text { apply } \\
\mathrm{Nr} \%\end{array}$}} & \multirow{2}{*}{\multicolumn{2}{|c|}{$\begin{array}{l}\text { Sometim } \\
\text { es apply } \\
\mathrm{Nr} \% \\
\end{array}$}} & \multirow{2}{*}{\multicolumn{2}{|c|}{$\begin{array}{r}\text { Apply } \\
\text { always } \\
\text { Nr. } \%\end{array}$}} \\
\hline Results & & & & & & \\
\hline observations & 3 & 33.3 & 1 & 11.11 & 5 & 55.56 \\
\hline self-asseasment & 0 & 00.0 & 8 & 80.00 & 2 & 20.00 \\
\hline $\begin{array}{c}\text { Indicator } \\
1.4 .\end{array}$ & \multicolumn{6}{|c|}{$\begin{array}{l}\text { The specialist applies knowledge to } \\
\text { play an advocacy role when } \\
\text { development is hindered in some } \\
\text { way by actively making positive } \\
\text { changes to the environment, the } \\
\text { curriculum and the nature of } \\
\text { relationships that support optimal } \\
\text { learning. }\end{array}$} \\
\hline Statements & \multicolumn{2}{|c|}{$\begin{array}{c}\text { Does not } \\
\text { apply } \\
\mathrm{Nr} \%\end{array}$} & \multicolumn{2}{|c|}{$\begin{array}{l}\text { Sometim } \\
\text { es apply }\end{array}$} & \multicolumn{2}{|c|}{$\begin{array}{l}\text { Apply } \\
\text { always }\end{array}$} \\
\hline observations & 2 & 22.2 & 2 & 22.22 & 5 & 55.56 \\
\hline self-asseasment & 0 & 00.0 & 2 & 20.00 & 8 & 80.00 \\
\hline
\end{tabular}


Table 32. Principle 2: Continuous participation in professional development activities improves the quality of practice.

\begin{tabular}{|c|c|c|c|c|c|c|}
\hline $\begin{array}{c}\text { Indicator } \\
2.1 .\end{array}$ & \multicolumn{6}{|c|}{$\begin{array}{l}\text { The specialist seeks to support and } \\
\text { reinforce practice through } \\
\text { professional development activities } \\
\text { (vocational training and staff } \\
\text { training under supervision, work in } \\
\text { vocational learners' communities, } \\
\text { etc.) in all aspects of this } \\
\text { Framework, including skills for } \\
\text { developing human relationships. }\end{array}$} \\
\hline Statements & \multirow{2}{*}{\multicolumn{2}{|c|}{$\begin{array}{l}\text { Does not } \\
\text { apply } \\
\text { Nr. } \%\end{array}$}} & \multirow{2}{*}{\multicolumn{2}{|c|}{$\begin{array}{l}\text { Sometim } \\
\text { es apply } \\
\text { Nr. } \%\end{array}$}} & \multicolumn{2}{|c|}{$\begin{array}{c}\text { Apply } \\
\text { always }\end{array}$} \\
\hline Results & & & & & & \\
\hline observations & 0 & 00.0 & 3 & 33.33 & 6 & 66.67 \\
\hline self-asgessment & 0 & 00.0 & 8 & 80.00 & 2 & 20.00 \\
\hline $\begin{array}{c}\text { Indicator } \\
2.2 \text {. }\end{array}$ & \multicolumn{6}{|c|}{$\begin{array}{l}\text { The specialist uses current } \\
\text { self-assessment and reflection } \\
\text { practices. }\end{array}$} \\
\hline Statements & \multicolumn{2}{|c|}{$\begin{array}{c}\text { Does not } \\
\text { apply }\end{array}$} & \multicolumn{2}{|c|}{$\begin{array}{l}\text { Sometim } \\
\text { es apply }\end{array}$} & \multicolumn{2}{|c|}{$\begin{array}{c}\text { Apply } \\
\text { always }\end{array}$} \\
\hline observations & 2 & 22.2 & 2 & 22.22 & 5 & 55.56 \\
\hline self-assessment & 1 & 10.0 & 8 & 80.00 & 1 & 10.00 \\
\hline $\begin{array}{c}\text { Indicator } \\
2.3 \text {. }\end{array}$ & \multicolumn{6}{|c|}{$\begin{array}{l}\text { The specialist is involved in } \\
\text { learning communities with other } \\
\text { people who work with the same or } \\
\text { similar children and families to } \\
\text { engage in in-depth dialogues about } \\
\text { their practice, its impact on children, } \\
\text { and how it can be improved to } \\
\text { support learning and developing } \\
\text { children. }\end{array}$} \\
\hline Statements & \multirow{2}{*}{\multicolumn{2}{|c|}{$\begin{array}{l}\text { Does not } \\
\text { apply } \\
\text { Nr. } \%\end{array}$}} & \multirow{2}{*}{\multicolumn{2}{|c|}{$\begin{array}{l}\text { Sometim } \\
\text { es apply } \\
\text { Nr. } \%\end{array}$}} & \multirow{2}{*}{\multicolumn{2}{|c|}{$\begin{array}{c}\text { Apply } \\
\text { always } \\
\text { Nr. } \%\end{array}$}} \\
\hline Results & & & & & & \\
\hline observations & 3 & 33.3 & 1 & 11.11 & 5 & 55.56 \\
\hline self-assessment & 1 & 10.0 & 1 & 10.00 & 8 & 80.00 \\
\hline $\begin{array}{l}\text { Indicator } \\
2.4 \text {. }\end{array}$ & \multicolumn{6}{|c|}{$\begin{array}{l}\text { The specialist develops strategies } \\
\text { for responding to the } \\
\text { unpredictability and insecurity } \\
\text { caused by rapid changes in society } \\
\text { as a whole, } 21 \text { st century family life } \\
\text { and early childhood care. }\end{array}$} \\
\hline Statements & \multicolumn{2}{|c|}{$\begin{array}{l}\text { Does not } \\
\text { apply }\end{array}$} & \multicolumn{2}{|c|}{$\begin{array}{l}\text { Sometim } \\
\text { es apply }\end{array}$} & \multicolumn{2}{|c|}{$\begin{array}{l}\text { Apply } \\
\text { always }\end{array}$} \\
\hline Results & \multicolumn{2}{|c|}{$\mathrm{Nr} . \quad \%$} & \multicolumn{2}{|c|}{ Nr. $\%$} & \multicolumn{2}{|c|}{ Nr. $\quad \%$} \\
\hline observations & 3 & 33.3 & 6 & 66.67 & 0 & 00.00 \\
\hline self-assessment & 0 & 00.0 & 9 & 90.00 & 1 & 10.00 \\
\hline $\begin{array}{c}\text { Indicator } \\
2.5 .\end{array}$ & \multicolumn{6}{|c|}{$\begin{array}{l}\text { The specialist demonstrates the } \\
\text { understanding that personal } \\
\text { problems and potential prejudices or } \\
\text { barriers can affect the relationship } \\
\text { with children and families and seek } \\
\text { professional counseling and further } \\
\text { training if necessary. }\end{array}$} \\
\hline Statements & \multicolumn{2}{|c|}{$\begin{array}{c}\text { Does not } \\
\text { apply }\end{array}$} & \multicolumn{2}{|c|}{$\begin{array}{l}\text { Sometim } \\
\text { es apply }\end{array}$} & \multicolumn{2}{|c|}{$\begin{array}{l}\text { Apply } \\
\text { always }\end{array}$} \\
\hline Results & \multicolumn{2}{|c|}{$\mathrm{Nr} . \quad \%$} & \multicolumn{2}{|c|}{ Nr. $\%$} & \multicolumn{2}{|c|}{ Nr. $\%$} \\
\hline observations & 2 & 22.2 & 7 & 77.78 & 0 & 00.00 \\
\hline self-assessment & 0 & 00.0 & 5 & 50.00 & 5 & 50.00 \\
\hline
\end{tabular}

\subsection{Priority area "Cross-sectoral cooperation"}

Table 33. Principle 1: Interaction and cooperation with other professionals and services in the same and / or different sectors, such as ensuring privacy, confidentiality and dignity of children and families, improving the quality of service delivery.

\begin{tabular}{|c|c|c|c|c|c|c|}
\hline $\begin{array}{c}\text { Indicator } \\
1.1 .\end{array}$ & \multicolumn{6}{|c|}{$\begin{array}{l}\text { The specialist provides information, } \\
\text { referrals and links to other family } \\
\text { services that they or the child need } \\
\text { while providing privacy, privacy } \\
\text { and dignity. }\end{array}$} \\
\hline Statements & \multicolumn{2}{|c|}{$\begin{array}{c}\text { Does not } \\
\text { apply }\end{array}$} & \multicolumn{2}{|c|}{$\begin{array}{l}\text { Sometim } \\
\text { es apply }\end{array}$} & \multicolumn{2}{|c|}{$\begin{array}{l}\text { Apply } \\
\text { always }\end{array}$} \\
\hline Results & \multicolumn{2}{|c|}{ Nr. $\%$} & \multicolumn{2}{|c|}{ Nr. $\%$} & \multicolumn{2}{|c|}{ Nr. $\%$} \\
\hline observations & 5 & 55.5 & 4 & 44.44 & 0 & 00.00 \\
\hline self-assessment & 1 & 10.0 & 0 & 00.00 & 9 & 90.00 \\
\hline $\begin{array}{c}\text { Indicator } \\
1.2 .\end{array}$ & \multicolumn{6}{|c|}{$\begin{array}{l}\text { The specialist carefully leads } \\
\text { accurate dossiers for all children and } \\
\text { their families. }\end{array}$} \\
\hline Statements & \multicolumn{2}{|c|}{$\begin{array}{c}\text { Does not } \\
\text { apply }\end{array}$} & \multicolumn{2}{|c|}{$\begin{array}{l}\text { Sometim } \\
\text { es apply }\end{array}$} & \multicolumn{2}{|c|}{$\begin{array}{l}\text { Apply } \\
\text { always }\end{array}$} \\
\hline Results & & \multicolumn{2}{|c|}{ Nr. $\%$} & \multicolumn{2}{|c|}{ Nr. $\%$} \\
\hline observations & 0 & 00.0 & 6 & 66.67 & 3 & 33.33 \\
\hline self-assessment & 1 & 10.0 & 0 & 00.00 & 9 & 90.00 \\
\hline $\begin{array}{c}\text { Indicator } \\
1.3 .\end{array}$ & \multicolumn{6}{|c|}{$\begin{array}{l}\text { The specialist regularly cooperates } \\
\text { with colleagues from other services, } \\
\text { respecting the position, } \\
\text { responsibility and expertise of the } \\
\text { other. }\end{array}$} \\
\hline Statements & \multicolumn{2}{|c|}{$\begin{array}{c}\text { Does not } \\
\text { apply }\end{array}$} & \multicolumn{2}{|c|}{$\begin{array}{l}\text { Sometim } \\
\text { es apply }\end{array}$} & \multicolumn{2}{|c|}{$\begin{array}{l}\text { Apply } \\
\text { always }\end{array}$} \\
\hline Results & \multicolumn{2}{|c|}{ Nr. \% } & \multicolumn{2}{|c|}{ Nr. $\%$} & \multicolumn{2}{|c|}{ Nr. $\%$} \\
\hline observations & 4 & 44.4 & 0 & 00.00 & 5 & 55.56 \\
\hline self-assessment & 0 & 00.0 & 1 & 10.00 & 9 & 90.00 \\
\hline $\begin{array}{c}\text { Indicator } \\
1.4 .\end{array}$ & \multicolumn{6}{|c|}{$\begin{array}{l}\text { The specialist critically analyzes his } \\
\text { / her own professional preferences, } \\
\text { prejudices, biases, judgments, and } \\
\text { practices that can deliberately or } \\
\text { unintentionally affect children and } \\
\text { families. }\end{array}$} \\
\hline $\begin{array}{l}\text { Statements } \\
\text { Results }\end{array}$ & \multicolumn{2}{|c|}{$\begin{array}{l}\text { Does not } \\
\text { apply } \\
\text { Nr. } \%\end{array}$} & \multicolumn{2}{|c|}{$\begin{array}{l}\text { Sometim } \\
\text { es apply } \\
\text { Nr. } \%\end{array}$} & \multicolumn{2}{|c|}{$\begin{array}{l}\text { Apply } \\
\text { always } \\
\text { Nr. } \%\end{array}$} \\
\hline observations & 4 & 44.4 & 0 & 00.00 & 5 & 55.56 \\
\hline self-assessment & 0 & 00.0 & 3 & 30.00 & 7 & 70.00 \\
\hline $\begin{array}{c}\text { Indicator } \\
1.5 .\end{array}$ & \multicolumn{6}{|c|}{$\begin{array}{l}\text { The specialist communicates and } \\
\text { discusses with other experts who } \\
\text { also provide services for children } \\
\text { under the age of three in order to } \\
\text { learn more about how to ensure a } \\
\text { smooth transition and coordination } \\
\text { between their own service and } \\
\text { others. }\end{array}$} \\
\hline Statements & \multicolumn{2}{|c|}{$\begin{array}{l}\text { Does not } \\
\text { apply }\end{array}$} & \multicolumn{2}{|c|}{$\begin{array}{l}\text { Sometim } \\
\text { es apply } \\
\text { Nr. } \%\end{array}$} & \multicolumn{2}{|c|}{$\begin{array}{l}\text { Apply } \\
\text { always }\end{array}$} \\
\hline observations & 4 & 44.4 & 3 & 33.33 & 2 & 22.22 \\
\hline self-assessment & o & 00.0 & 8 & 80.00 & 2 & 20.00 \\
\hline $\begin{array}{c}\text { Indicator } \\
1.6 .\end{array}$ & & $\begin{array}{l}\text { spec: } \\
\text { denti } \\
\text { t the } \\
\text { oses } \\
\text { en pe } \\
\text { the cl }\end{array}$ & & $\begin{array}{l}\text { maint } \\
\text { of a1 } \\
\text { and } t \\
\text { ly if th } \\
\text { sion fro } \\
\text { at risk }\end{array}$ & & $\begin{array}{l}\text { mplete } \\
\text { ilyation and } \\
\text { explicit } \\
\text { parents }\end{array}$ \\
\hline Statements & $\mathrm{N}$ & $\begin{array}{l}5 \text { not } \\
\%\end{array}$ & & $\begin{array}{l}\text { metim } \\
\text { apply } \\
\%\end{array}$ & & $\begin{array}{l}\text { pply } \\
\text { ways } \\
\%\end{array}$ \\
\hline observations & 3 & 33.3 & 6 & 66.67 & 0 & 00.00 \\
\hline self-assessment & 0 & 00.0 & 1 & 10.00 & 9 & 90.00 \\
\hline $\begin{array}{c}\text { Indicator } \\
1.7 .\end{array}$ & & $\begin{array}{l}\text { spec } \\
\text { colle } \\
\text { iders } \\
\text { he ad } \\
\text { isive } \\
\text { youn }\end{array}$ & & $\begin{array}{l}\text { supp } \\
\text { s and } \\
\text { ow be: } \\
\text { n of d } \\
\text { aches } \\
\text { dren. }\end{array}$ & & $\begin{array}{l}\text { lalogue } \\
\text { service } \\
\text { lvocate } \\
\text { tic and } \\
\text { ces for }\end{array}$ \\
\hline Statements & $\mathbf{N}$ & $\begin{array}{l}\text { s not } \\
\text { ply } \\
\%\end{array}$ & & $\begin{array}{l}\text { metim } \\
\text { apply } \\
\%\end{array}$ & & $\begin{array}{l}\text { Pply } \\
\text { vays } \\
\%\end{array}$ \\
\hline observations & 4 & 44.4 & 5 & 55.56 & 0 & 0.00 \\
\hline self-assessment & 7 & 70.0 & 1 & 10.00 & 2 & 20.00 \\
\hline
\end{tabular}


Table 34. Principle 2: Consultation with an Early Child Diagnostic Specialist for formal screening and assessment where necessary prevents multiple developmental delays and atypical behaviors later in life.

\begin{tabular}{|c|c|c|c|c|c|c|}
\hline $\begin{array}{c}\text { Indicator } \\
2.1 \text {. }\end{array}$ & \multicolumn{6}{|c|}{$\begin{array}{l}\text { The specialist documents } \\
\text { observations to help early childhood } \\
\text { specialists identify children who } \\
\text { may have delayed development or } \\
\text { disability. }\end{array}$} \\
\hline Statements & \multicolumn{2}{|c|}{$\begin{array}{c}\text { Does not } \\
\text { apply }\end{array}$} & \multicolumn{2}{|c|}{$\begin{array}{l}\text { Sometim } \\
\text { es apply }\end{array}$} & \multicolumn{2}{|c|}{$\begin{array}{l}\text { Apply } \\
\text { always }\end{array}$} \\
\hline Results & \multicolumn{2}{|c|}{ Nr. $\%$} & \multicolumn{2}{|c|}{ Nr. $\quad \%$} & \multicolumn{2}{|c|}{ Nr. $\quad \%$} \\
\hline observations & 1 & 11.1 & 6 & 66.67 & 2 & 22.22 \\
\hline self-assessment & 1 & 10.0 & 6 & 60.00 & 3 & 30.00 \\
\hline $\begin{array}{c}\text { Indicator } \\
2.2 \text {. }\end{array}$ & \multicolumn{6}{|c|}{$\begin{array}{l}\text { The specialist uses valid, reliable } \\
\text { and appropriate screening tools } \\
\text { recommended by early childhood } \\
\text { diagnostics to monitor the condition } \\
\text { of children who are found to be } \\
\text { lagging behind, have disabilities and } \\
\text { atypical behaviors. }\end{array}$} \\
\hline Statements & \multicolumn{2}{|c|}{$\begin{array}{c}\text { Does not } \\
\text { apply }\end{array}$} & \multicolumn{2}{|c|}{$\begin{array}{l}\text { Sometim } \\
\text { es apply }\end{array}$} & \multicolumn{2}{|c|}{$\begin{array}{l}\text { Apply } \\
\text { always }\end{array}$} \\
\hline Results & \multicolumn{2}{|c|}{ Nr. $\%$} & \multicolumn{2}{|c|}{ Nr. $\%$} & \multicolumn{2}{|c|}{ Nr. $\quad \%$} \\
\hline observations & 8 & 88.8 & 1 & 11.11 & 0 & 00.00 \\
\hline self-assessment & 7 & 70.0 & 1 & 10.00 & 2 & 20.00 \\
\hline $\begin{array}{c}\text { Indicator } \\
2.3 \text {. }\end{array}$ & \multicolumn{6}{|c|}{$\begin{array}{l}\text { The specialist is open and } \\
\text { collaborates with early childhood } \\
\text { specialists to apply the proposed } \\
\text { activities to children who are found } \\
\text { to have developmental or disability } \\
\text { impairment. }\end{array}$} \\
\hline Statements & \multicolumn{2}{|c|}{$\begin{array}{c}\text { Does not } \\
\text { apply }\end{array}$} & \multicolumn{2}{|c|}{$\begin{array}{l}\text { Sometim } \\
\text { es apply }\end{array}$} & \multicolumn{2}{|c|}{$\begin{array}{l}\text { Apply } \\
\text { always }\end{array}$} \\
\hline Results & \multicolumn{2}{|c|}{ Nr. \% } & \multicolumn{2}{|c|}{ Nr. $\%$} & \multicolumn{2}{|c|}{ Nr. $\quad \%$} \\
\hline observations & 1 & 11.1 & 8 & 88.89 & 0 & 00.00 \\
\hline self-assessment & 0 & 00.0 & 8 & 80.00 & 2 & 20.00 \\
\hline $\begin{array}{c}\text { Indicator } \\
2.4 \text {. }\end{array}$ & \multicolumn{6}{|c|}{$\begin{array}{l}\text { The specialist is careful when } \\
\text { talking to parents and families about } \\
\text { visiting early childhood diagnostics, } \\
\text { or about slowing down development } \\
\text { or disability. }\end{array}$} \\
\hline Statements & \multicolumn{2}{|c|}{$\begin{array}{l}\text { Does not } \\
\text { apply }\end{array}$} & \multicolumn{2}{|c|}{$\begin{array}{l}\text { Sometim } \\
\text { es apply }\end{array}$} & \multicolumn{2}{|c|}{$\begin{array}{l}\text { Apply } \\
\text { always }\end{array}$} \\
\hline observations & 1 & 11.1 & 8 & 88.89 & 0 & 00.00 \\
\hline self-assessment & 0 & 00.0 & 1 & 10.00 & 9 & 90.00 \\
\hline $\begin{array}{c}\text { Indicator } \\
2.5 \text {. }\end{array}$ & \multicolumn{6}{|c|}{$\begin{array}{l}\text { The specialist draws and relies on } \\
\text { parent/family information as part of } \\
\text { the ongooing evaluation process } \\
\text { when working with children with } \\
\text { developmental or disability } \\
\text { impairment. }\end{array}$} \\
\hline Statements & \multicolumn{2}{|c|}{$\begin{array}{l}\text { Does not } \\
\text { apply }\end{array}$} & \multicolumn{2}{|c|}{$\begin{array}{l}\text { Sometim } \\
\text { es apply }\end{array}$} & \multicolumn{2}{|c|}{$\begin{array}{l}\text { Apply } \\
\text { always }\end{array}$} \\
\hline observations & 2 & 22.2 & 6 & 66.67 & & 11.11 \\
\hline self-assessment & 0 & 00.0 & 7 & 70.00 & 3 & 30.00 \\
\hline $\begin{array}{c}\text { Indicator } \\
2.6 \text {. }\end{array}$ & & $\begin{array}{l}\text { speci } \\
\text { can } \\
\text { ial te } \\
\text { hood } \\
\text { denti }\end{array}$ & & $\begin{array}{l}\text { uses th } \\
\text { obtaine } \\
\text { conduct } \\
\text { pecialis }\end{array}$ & & $\begin{array}{l}\text { rmation } \\
\text { in the } \\
\text { in early } \\
\text { in a }\end{array}$ \\
\hline Statements & $\begin{array}{r}\mathrm{Dc} \\
\mathrm{a} \\
\mathrm{Nr} .\end{array}$ & $\begin{array}{l}\text { not } \\
\% \\
\%\end{array}$ & & $\begin{array}{r}\text { metim } \\
\text { apply } \\
\%\end{array}$ & & $\begin{array}{l}\text { pply } \\
\text { ways } \\
\% \\
\end{array}$ \\
\hline oservations & 3 & 33.3 & 6 & 66.67 & 0 & 00.00 \\
\hline self-assessment & 0 & 00.0 & 7 & 70.00 & 3 & 30.00 \\
\hline
\end{tabular}

\section{DISCUSSIONS}

Identified as the first priority in the "Quality Framework for Early Childhood Services" (QFECS), Area 1 "Relationships" emphasizes that social relations are at the heart of the development of children under the age of three. The principles of adjoining indicators laid down in this area affirm that "the enrichment of children's relationships and emotional experiences, the absorption of social norms, the stimulation of self-awareness are basic structural components of the child's social development" (Derrijan and Valchev, 2015: 36).

The presented results of the surveyed priority area reveal that responsive, equitable and equal interactions have been developed in the Jesuit groups under investigation. The high degree of their manifestation is revealed by the statements made in the Daily Self-Assessment Report of the members of the cluster teams participating in the study.

Relatively close, the values of observations and self-assessment in the column "Applying permanently" are an expression of the conscious aspirations of professionals to promote relationships with the child that support their initiative in the process of communication - see "Relationships": Principle 1, Indicators 1.2 , 1.5; Principle 2, Indicators 2.1, 2.3, 2.4; Principle 3, Indicators 3.2, 3.4; Principle 5, Indicators 5.1, 5.2.

The results that reflect the highlighted statements in the "Partially applied" column should also be disregarded. Their presence is an indicator that definitely signals the still dominant attitudes of some of the team's cluster representatives that their role is only related to meeting the underlying needs of the child in the middle age. This observation highlights that institutional care professionals need targeted work to promote the under-standing that "the earliest social task is at-tachment" (Tasevska, 2014: 39).

The indicators in Area 2 "Family and Community" focus on the quality of early childhood services that ensure a climate of tolerance and dialogue between the institution's and the family's representatives.

The results of the survey show that monitoring scores compared to self-assessment of crèche representatives overlap to a high degree - see Principle 2 "Sensitive, respectful, and equal family communication supports the development and learning of children."

By itself, this fact reveals the already formed attitudes of the representatives of the cluster teams to communicate with families in 
ways that show respect and appreciation, as well as to jointly discuss all decisions about the development, well-being and learning of their children.

The results reflecting Principle 3 "Services are best provided in partnership with the family" and Principle 4 "Partnerships based on cooperative and community-based cooperation best support families and children" reveal discrepancies in the "Daily Surveillance Report" and in the "Daily self-assessment report". Their analysis is the basis for the following conclusions:

- Clearly cluster teams take action to implement an institutional policy related to the transformation of "working with the family" into "interacting with parents." We find a testimony in the highlighted responses to the Families and Community indicators, Principle 3, Indicators 3.1, 3.2, 3.4, 3.5;

- there are still no practices that ensure "safe transition" where specialists can "negotiate with different services to support families" - see Family and Community, Principle 4, Indicator 4.1;

- despite the theoretical preparedness of the cluster staff, there is still insufficient use of "opportunities for members of the community involvement and participation in early childhood services "- see Family and Community, Principle 4, Indicators 4.2 and 4.3.

This task "is complicated by the fact that an individual approach is necessary, which in turn makes the standardization method virtually impossible" (Biletska, 2017: 66), ie the theoretical preparedness of the specialists depends on the level of their professional reflexive competence.

Undoubtedly, the Principles in the Third Priority Area "Inclusiveness, Diversity and Values of Democracy", correspond directly to the indicators of the first and second priorities of the "Quality Framework for Early Childhood Services" (QFECS).

Confessing the values of democracy by all actors in the childcare care interactions up to the age of 3 are a strong indicator of a change in the provision of services in crèches.

The qualitative analysis of responses from the Third Priority Area reveals the following more significant summaries:

- the prevailing answers in the "Partial Apply" and "Applying Permanently," both marked in both the Daily Surveillance Report and the Daily Re-Port for Self-Assessment, reveal the unbalanced implementation of activities that ensure the promotion of the right of every child and his /her family be included, respected, appreciated - see area "Inclusion, diversity and values of democracy", Principle 1 , Indicators 1.1, 1.3, 1.4; Principle 2, Indicators $2.1,2.3,2.4$; Principle 3 , indicators 3.2, 3.3, 3.5; Principle 4, Indicators 4.1, 4.3, 4.4; - the identified partiality in this direction unambiguously reveals the need to upgrade the competencies of the cluster teams in areas that are definitely important for enhancing the quality of early childhood services - see "Inclusion, Diversity and Values of Democracy," Principle 2, Indicators 2.1, 2.2, 2.3; Principle 3 , indicators 3.2, 3.3, 3.5; Principle 4, Indicators 4.1, 4.3, 4.4;

- it is positively commented on the fact that they are marked in this way (mostly in the "Partially applied" and "Applying permanently" scales), the responses to the study reveal the "awareness" of the key weight of the period from 0 to 3 years for the development of all the identities of the child - see area "Inclusion, diversity and values of democracy", Principle 3, Indicators 3.1, 3.2, 3.3 and 3.4.

It can be summed up that the results of the monitoring program in this priority area reveal the readiness of the clergy specialists to formulate clearer organizational messages regarding their professional inclusion in the social and emotional adaptation of the children of the elderly.

At first reading, the Fourth Priority Area of the "Quality Framework for Early Childhood Services" (QFECS) suggested results that would not give rise to any doubts about the quality of health, well-being and nutrition services for children under 3 years of age.

A deeper analysis of the survey results reveals inconsistencies in the marked responses from the monitoring and the members of the cluster teams.

In fact, "Claiming Health" is the most consistently highlighted in applying Principle 1 "Every child's health is being promoted" - a result that is reflected in Indicators 1.1, 1.2, $1.3,1.4,1.5,1.6,1.7,1.8$.

The results recorded in Principle 2, Principle 3 and Principle 4 show fluctuations in the extent to which they are applied: they mainly vary between "Partially applied" and "Not applicable" (see area 4 - Principle 2, Indicators 2.1, 2.2, 2.3 , 2.4, 2.5, 2.6, Principle 3, Indicators $3.1,3.3,3.4,3.5$, Principle 4, Indicators 4.1, 4.2, 4.3 and 4.4).

There are also contradictions in the results reported in the Daily Survey Survey and the Daily Self-Assessment Report - Principle 2, Indicators 2.1, 2.2, 2.3, 2.4, 2.6; Principle 3, 
Indicators 3.1, 3.3, 3.4 and Principle 4, Indicator 4.2 .

These contradictions focus attention on the need to rethink the process of interaction towards the understanding that:

- health and nutrition with children under the age of 3 are an integral part of creating affection;

- the affection of the child under the age of 3 is a dynamic process that requires the continuous creation of close and positive interactions during nutrition and care.

An important part of supporting children under the age of 3 is to master procedures for giving recommendations to families when children show signs of atypical behavior, depression or anxiety. The analysis of the results in this context unambiguously shows that "joint engagement in this process requires, besides recognition and acceptance, also readiness to discuss differences of opinion and to deal with everyday problems" (Vitanova and Miteva, 2017: 201)

Undoubtedly, the Fifth Priority Area is extremely important in the Quality Framework for Early Childhood Services. Development and learning are the synchronized process that allows the potential of the child under the age of 3 to be unleashed.

The marked responses in the "Daily report for self-assessment" are mostly in the highest scale of the "Applying permanently" scale - see Development and Learning, Principle 1, Indicators 1.1, 1.2, 1.3, 1.4; Principle 2, Indicators 2.1, 2.4, 2.5; Principle 3, Indicators $3.1,3.2,3.4,3.5$; Principle 4, Indicators 4.1, 4.2, 4.3. 4.4.

On the other hand, the dynamics in the responses from the "Daily Surveillance Report" deprives the credibility of the registered indicative components by the cluster teams, given that the monitoring expert predominantly notes the claims in the "Partially applied" and "Not applicable" columns - see Development and Learning Area, Principle 1, Indicators 1.1, 1.3, 1.4; Principle 2, Indicators 2.1, 2.2, 2.3, 2.4, 2.5, 2.6; Principle 3, Indicators $3.3,3.4,3.5$; Principle 4, indicators 4.1, 4.2, $4.3,4.4$.

This finding draws attention to a relatively high percentage amongst the cluster members who are sufficiently aware that care must always be approached as opportunities.

Nevertheless, it turns out that practitioners do not have the specific competencies necessary for "situations in which, when playing, the child exhibits his abilities and personal qualities, inspired by the experience and gain- ing on his basis new own experience" (Dimitrova, 2017: 33) to make the most of them for development, learning and well-being for the child under 3 years of age.

"Surveillance and Documentation" (Sixth Priority Area in R \& DD) is the basis for adults to be open and to build relationships with babies and very young children.

There is a growing recognition that systematic "monitoring and documentation" of the well-being of children under the age of 3 determines the planning of new learning practices, the adaptation of the environment as well as the refinement of timetables, activities and adaptation of care needed for early childhood development.

These regularities underline the importance of the Sixth Priority Area in providing quality services in early childhood. There are again inconsistencies in the classification of these statements.

Representatives of the cluster teams have their answers mostly in the "Applying permanently" scale against the "Part-Apply" and "Non-Applicable" responses marked by the Expert-Monitoring - see Section 6, Principle 1, Indicators 1.1, 1.3, 1.4; Principle 2, Indicators 2.1, 2.2, 2.3, 2.4; Principle 3, Indicators 3.1, 3.2, 3.3, 3.4; Principle 4, indicators 4.1, 4.2 and 4.3.

It can be summed up that data in the "Daily Surveillance Report" and in the "Daily Self-Assessment Report" reveals the objectively binding of the observed indicator components in the marked answers. Their analysis is in the direction of the different starting points that respondents place in the focus of their attention:

- Certainly the representatives of the cluster teams, in the capacity of medical specialists, carry out systematic observations and document the actual health status of each child, which explains the claims in the degree "Applying permanently";

- as a pedagogical specialist, the monitoring expert focuses on the essentials in the documentation and planning process: as a way to take into account the progress of the child in the interactions that explains the claims in the "Partial Apply" and "Not Applicable" assumptions.

It is objectively appreciated that positive changes are reported in the efforts of the cluster teams to respond to the need for close relationships with parents. A testimony in this direction is the sharing of analyzes of the instrument used to evaluate child achievement - the child's portfolio - used in the three clergy 
groups.

Undoubtedly, the Seventh priority area of the "Quality Framework for Early Childhood Services" (QFECS) has accumulated the highest number of matches in the registered results from the cluster teams and the monitoring expert. The observed similarities of the reported statements are observed in the separate 5 principles and their corresponding indicators.

Since the indicators of a safe, healthy and supportive environment are meaningful, the high values of the positive response are a clear indicator of the validation of the appropriate choice in the organization of the environment in the study groups.

This result corresponds directly to the quality of services in the early childhood and is a reliable source for the following summaries:

- cluster teams maintain a physical and emotional environment that minimizes conflicts among young children - see Priority Area "Supporting Circles", Principle 1, Indicators 1.1, 1.2, 1.3, 1.4, 1.5;

- cluster teams provide sufficient resources to stimulate all areas of child development - see Priority Area "Supporting Circles", Principle 4, Indicators 4.2 and 4.3;

- the blind teams are aware of the crucial role of a supportive environment that develops a sense of belonging to the child up to the age of 3 , Principle 5, indicator 5.2.

The complexity and complexity of the indicators under discussion in this priority area requires the following statement: additional care is needed for the specialists in the cleric groups to use appropriate pedagogical interventions in the course of interactions with the child under the age of 3 (see Seventh area, Principle 2, Indicator 2.3, Principle 3, Indicators 3.1, 3.4, Principle 5, Indicators 5.1, 5.3).

In this direction for the pedagogy of early childhood there is a sufficiently large array of studies devoted to the psychological characteristics of children. It is their age-specificity that argued that "authoritarian educational methods and normative directives a priori can not be dominant at this age and can not determine the child's invisible and visible selfesteem" (Yanakieva, 2014).

The principles included in the Eighth Priority Area focus on the link between the quality of services provided by children under the age of three and the level of training and professional development of adults working with them. The indicators that "illuminate" this relationship are sufficiently eloquent to reflect the current state of the respondents in this study.

Coincidences in the claims marked in the Daily Surveillance Report and in the Daily Self-Assessment Report are clearly noticed see "Professional Development", Principle 1, Indicator 1.1, 1.2; Principle 2, Indicator 2.1, $2.2,2.3$, which reveals the attitudes of the teams scrutinized to increase their competencies towards addressing the specific needs of the child under the age of 3 .

However, the reported contradictions in Principle 1, Indicators 1.3, 1.4 and Principle 2, Indicators $2.4,2.5$ - a finding pointing to the fact that there are still some specialists accompanying children under the age of 3 who "Do not think that it is necessary to continually analyze and enrich their own teaching experience" (Stošić and Stošić, 2013).

It is obvious that adults working with children under 3 years need a toolbox that:

- allows identification of main areas and experience in the child's holistic development in order to communicate sensitively with families;

- requires an advocacy role when development of the child is hindered in any way; help develop strategies to respond to the unpredictability and insecurity caused by rapid changes in society as a whole in family life in the $21^{\text {st }}$ century.

The 9" $^{\text {th }}$ priority Area "Cross-sectoral cooperation" draws attention to the need to apply the multidisciplinary approach in early childhood. Naturally, this process needs knowledge that goes some way beyond the professional competence of adults accompanying a child from 0 to 3 years.

The results in the attached tables (No. 32 and No. 33) outline the tendency for sufficiently active and searching behavior of the teams of the groups in this direction. The presented ratios are a demonstration of the maturity and responsibility of the participants about the irreversible sensitivity of the age period and the maximum utilization of the internal conditions for development of the child created by this sensitivity.

Registered answers in the "Partially applied" and "Not Applicable" roles - see Nine Fields, Principle 1, Indicators 1.1, 1.4, 1.6, 1.7; Principle 2, indicators 2.2, 2.3. 2.4, 2.5 and 2.6, reveals the difficulty of the cluster teams:

- to provide care and support services to children and their families during critical early childhood periods;

- to use valid, reliable and appropriate 
screening tools recommended by early childhood diagnostics to monitor the condition of children;

- to maintain a trust relationship with the families of children with developmental delays, in order to obtain up-to-date information as part of the ongoing evaluation process; - to ensure a smooth transition in the establishment of a network of relations to ensure partnership with institutions and non-governmental organizations (NGOs).

\section{CONCLUSIONS}

The results of the study, which is related to the issue of early childhood well-being in the institutional care system, reveal deficiencies resulting from:

- applying still inertial patterns of interactions with children and their families;

- unjustified, partial and therefore ineffective structuring of a unified strategy for the functioning of the CSS;

- sporadic, partial and fragmentary attempts to transform institutional care policies into early childhood.

Undoubtedly, the principles and indicators differentiated in the Nine Priority Areas of the Applied Framework outline the essence of the relationship and meaning of care with children from birth to 3 years. Formulated metrics make it possible to specify the steps in the process of their creation, deployment and maintenance. It can be conclusively concluded that its use impersonates "the necessity for educational results in the shape of competence as an indicator of human capital" (Tsankov, 2018: 69).

In this regard, the objective analysis of the results obtained from the "Quality Framework for Early Childhood Services" (QFECS) in the three clerical groups reveals a priori its reflected reflexion, causing proactive (conscious) striving for quality services at all levels. It is precisely this inductive feature that nominates the Framework as a reliable and robust tool for enhancing the quality of early childhood services.

\section{ACKNOWLEDGEMENTS}

The presented study was carried out as part of an international study on the implementation of the "Quality Framework for Early Childhood Services" initiated by the ISSA (International Steps by Step) Association.

\section{Conflict of interests}

The author declares no conflict of interest.

\section{REFERENCES}

Biletska, M. (2017). Principi j metodologichni pidhodi do rozvitku pam'iati $\mathrm{v}$ navchalsnij diialsnosti [Principles and methodological approaches to the memory development in educational activities]. Scientific herald of Melitopol State Pedagogical University. Series: Pedagogy. No 1. p. 60-67. http://dx.doi.org/10.7905/nvmdpu. v0i18.1890

Dyankova, G. (2018a). Tolerantnost v delnichnost i praznichnost. Metodicheski algoritym za neformalno obrazovanie i inovativni profesionalni umeniia na uchitelia za mezhduetnicheska tolerantnost $\mathrm{v}$ detskata gradina. [Tolerance in daytime and festivity, methodical algorithm for nonformal education and innovative professional skills for interethnic tolerance in the kindergarten]. Farago, Sofia, p. 96.

Dyankova, G. (2018b). Research of cognitive exchange Dyankova, G. (2018). Research of cognitive exchange specifics in teachers academic training. International Journal of Cognitive Research in Science, Engineering and Education, 6(3). https://doi.org/10.5937/ijcrsee1803001D

Derijan, I. \& Valchev, G. (2015). Determinanti na deficitarnoto razvitie pri deca, lisheni ot roditelski grizhi. [Determinants of deficits in the development in children deprived of parental care], BSU, Center for Humanities, issue 1, pp.3545. Retrieved from http://research.bfu.bg:8080/ jspui/handle/123456789/727

Dimitrova, Zl. (2017). Proekcii na animativniia podhod $\mathrm{v}$ pedagogikata. [Projections of the animation approach in pedagogical sciense], Education and tehnologies, 8, pp. 33-37. Retrieved from http://www.edutechjournal.org/wp-content/uploads/2017/09/8_1_33-37.pdf

EC-European Commission. (2011). Early childhood education and care: providing all our children with the best start for the world of tomorrow. Brussels, European Commission [COM (2011) 66 final], available at: http://ec.europa.eu/assets/eac/education/policy/strategic-framework/ archive/documents/ecec-quality-framework en.pdf

European commission (2014). Early childhood education and care 2014. [Eurydice Policy Brief], Education, Audiovisual and Culture Executive Agency, p. 34. https://publications.europa.eu/ en/publication-deail/-/publication/25c63f11c2cb-4c4c-a3cb-85d9b1 eab93b/language-en/ format-PDF/source-search

European commission (2011), COMMUNICATION FROM THE COMMISSION Early Childhood Education and Care: Providing all our children with the best start for the world of tomorrow, [European commission, Brussels, 17.02.2011], p.11. https://eur-lex.europa.eu/LexUriServ/LexUriServ.do?uri=COM:2011:0066:FIN:EN:PDF

Green, B., C. Ayoub, J. DymBartlett, A. Ende, C. Furrer, R. Chazan-Cohen, C. Vallotton, J. Klevens (2014). The effect of Early Head Start on child welfare system involvement: A first look at lon- 
gitudinal child maltreatment outcomes, Children and Youth Services Review, 42, July 2014, Pages 127-135. https://doi.org/10.1016/j.childyouth.2014.03.044

Kaleynska, T. (2014). Children and Inclusion - European standards and community implementation. Challenges in Building Child Friendly Communities. University of Osijek and Evropski dom, Slavonski Brod, Zadar, Croatia, pp. 124 130. Retrieved from http://da.uni-vt.bg/pubinfo. aspx? $\mathrm{p}=11182$

Mihova, M. (2012). Preduchilishtnoto obrazovanie prez prizmata na evropejskata i nacionalnata obrazovatelna politika. [Preschool education in the light of european and national policy], 130 years of pre-school education in Bulgaria, University Publishing House St. St. Cyril and Methodius, Veliko Tyrnovo, pp.122-127. Retrieved from http://da.uni-vt.bg/pubinfo.aspx? $\mathrm{p}=13321$

OECD (2012). Starting Strong III - A Quality Toolbox for Early Childhood Education and Care, http:// www.oecd.org/education/school/startingstrogiii-aqualitytoolboxforearlychildhoodeducationandcare.htm

Stošić, L., \& Stosic, I. (2013). Diffusion of innovation in modern school. International Journal of Cognitive Research in Science, Engineering and Education:(IJCRSEE), 1(1), 5-13. Retrieved from http://www.ijcrsee.com/index.php/ijcrsee/ article/view/219

Tasevska, D. (2008). SOS Semejstva v kriza, psihologicheska podkrepa na disfunkcionalni semejstva za efektivno roditelstvo. [SOS Families in crisis, psychological support of dysfunctional families for effective parenting]. University Publishing House St. St. Cyril and Methodius, Veliko Tyrnovo, p. 12.

Tasevska, D. (2014). Obrazovanie za ravni prava. [Education for Equal Rights]. University Publishing House St. St. Cyril and Methodius, Veliko Tyrnovo, p. 39

Tsankov, N. (2018). The transversal competence for problem-solving in cognitive learning. International Journal of Cognitive Research in Science, Engineering and Education, 6(3). pp. 67-82. https://doi.org/10.5937/ijcrsee1803067T

Vitanova, N. \& Miteva, N. (2017) Team teaching i/ili Co-teaching $\mathrm{v}$ konteksta na priobshtavashtoto $\mathrm{i}$ interkulturnoto obrazovanie. [Team Teaching and/or Co-Teaching in the Context of Inclusion and Intercultural Education], Strategies for Policy in Science and Education, 25(2) pp.190-208. Retrieved from https://azbuki. bg/editions/journals/329-staregies/sonparticles2016-2/2882-

Yanakieva, E. (2014) Detskoe schastьe kak pedagogicheskaia cennostь [Children's happiness as pedagogical value], A science. Thought: electronic periodic journal. Retrieved from https:// cyberleninka.ru/article/n/detskoe-schastie-kakpedagogicheskaya-tsennost-1 